JOURNAL OF THE

AMERICAN MATHEMATICAL SOCIETY

Volume 21, Number 4, October 2008, Pages 1019-1063

S 0894-0347(07)00579-6

Article electronically published on November 29, 2007

\title{
STABLE SELF-SIMILAR BLOW UP DYNAMICS FOR THE THREE DIMENSIONAL RELATIVISTIC GRAVITATIONAL VLASOV-POISSON SYSTEM
}

\author{
MOHAMMED LEMOU, FLORIAN MÉHATS, AND PIERRE RAPHÄ̈L
}

\section{Introduction}

1.1. Setting of the problem. Consider the $N$ dimensional gravitational VlasovPoisson system, with $N=3$ or 4 :

$$
(V P)\left\{\begin{array}{l}
\partial_{t} f+v \cdot \nabla_{x} f-E_{f} \cdot \nabla_{v} f=0, \quad(t, x, v) \in \mathbb{R}_{+} \times \mathbb{R}^{N} \times \mathbb{R}^{N}, \\
f(t=0, x, v)=f_{0}(x, v) \geq 0,
\end{array}\right.
$$

where we denoted for a given distribution function $f \geq 0$ :

$$
E_{f}(x)=\nabla_{x} \phi_{f}, \quad \phi_{f}(x)=-\frac{1}{(N-2) \sigma_{N}} \frac{1}{|x|^{N-2}} \star \rho_{f}, \quad \rho_{f}(x)=\int_{\mathbb{R}^{N}} f(x, v) d v,
$$

and $\sigma_{N}$ is the area of the unit sphere in $\mathbb{R}^{N}\left(\sigma_{3}=4 \pi\right.$ and $\left.\sigma_{4}=2 \pi^{2}\right)$. This nonlinear transport equation describes in dimension $N=3$ the mechanical state of a stellar system subject to its own gravity (see for instance [3, 14]). Classical calculations show that this model should be correct only for low velocities, and if high velocities occur, special relativistic corrections should be introduced; see Van Kampen and Felderhof [4, Glassey and Schaeffer [18] and references therein. A more accurate model is then provided by the three dimensional relativistic gravitational VlasovPoisson system:

$$
(R V P)\left\{\begin{array}{l}
\partial_{t} f+\frac{v}{\sqrt{1+|v|^{2}}} \cdot \nabla_{x} f-E_{f} \cdot \nabla_{v} f=0, \quad(t, x, v) \in \mathbb{R}_{+} \times \mathbb{R}^{3} \times \mathbb{R}^{3}, \\
f(t=0, x, v)=f_{0}(x, v) \geq 0
\end{array}\right.
$$

with $E_{f}$ given by (1.2). Note that the defocusing counterpart of (1.3) obtained by changing the sign in the nonlinearity $E_{f}$ appears in plasma physics as a simplified model to the full relativistic Vlasov-Maxwell system; see for example [17, 19, 20, 21, 22, 10, 11. The ultra-relativistic limit of (1.3) would lead one to consider the ultra-relativistic gravitational Vlasov-Poisson system:

$$
(U R V P)\left\{\begin{array}{l}
\partial_{t} f+\frac{v}{|v|} \cdot \nabla_{x} f-E_{f} \cdot \nabla_{v} f=0, \quad(t, x, v) \in \mathbb{R}_{+} \times \mathbb{R}^{3} \times \mathbb{R}^{3} \\
f(t=0, x, v)=f_{0}(x, v) \geq 0
\end{array}\right.
$$

Received by the editors November 8, 2006.

2000 Mathematics Subject Classification. Primary 82C70, 35Q55, 35Q75, 85A05, 74H35.

Key words and phrases. Vlasov-Poisson equations, relativistic gravitational kinetic equations, blow-up dynamics, virial law, modulation theory. 
These models belong to a hierarchy of physical models which aim at taking into account sharper gravitational effects. A complete relativistic model would require taking into account the full Vlasov-Einstein system; see for example [1], 9]. Note that the ultrarelativistic case (1.4) is related to what is described in physics literature as massless particles which have been considered in various situations in relativistic models; see for example Dafermos 8, Martin-Garcia and Gundlach 31, Choquet-Bruhat and Noutchegueme [6].

From the mathematical point of view, the local Cauchy problem for the VlasovPoisson system (1.1) and the relativistic Vlasov-Poisson system (1.3) is still not well understood. The existence of local in time weak solutions in the natural energy space is known, but uniqueness of the weak solution is a major open problem; see for example the books of Bouchut, Golse, Pulvirenti [4] and of Glassey [17] and references therein. Nevertheless, a satisfactory theory of strong solutions is known from the works of Pfaffelmoser [37, Lions and Perthame [30, Schaeffer 42] for the nonrelativistic case (1.1), and Glassey and Schaeffer [18] for the relativistic case (1.3). Note that this last work is restricted to radially symmetric initial data that is depending only on $(|x|,|v|, x \cdot v)$. We may summarize these results in the following statement. Let us denote for $k=0,1$ :

$\mathcal{C}_{0, \text { rad }}^{k}=\left\{f: \mathbb{R}^{2 N} \rightarrow[0,+\infty)\right.$ with radial symmetry, compactly supported and $\left.\mathcal{C}^{k}\right\} ;$ then

Theorem 1 (Local well posedness of classical solutions). Let $f_{0} \in \mathcal{C}_{0, \text { rad }}^{1}$; then there exists $T>0$ and a unique maximal solution

$$
f \in \mathcal{C}^{1}\left([0, T), \mathcal{C}_{0, \text { rad }}^{0}\right) \cap \mathcal{C}^{0}\left([0, T), \mathcal{C}_{0, \text { rad }}^{1}\right)
$$

to (1.1) and (1.3). Moreover, if $T<+\infty$, then

$$
\begin{gathered}
\text { for (1.1), }\left.\left.\quad|| v\right|^{2} f(t)\right|_{L^{1}} \rightarrow+\infty \quad \text { as } t \rightarrow T, \\
\text { for (1.3) }, \quad\left|\sqrt{1+|v|^{2}} f(t)\right|_{L^{1}} \rightarrow+\infty \quad \text { as } t \rightarrow T .
\end{gathered}
$$

Remark 1. Note in particular that the solution $f$ is nonnegative, and in this paper we shall only deal with such nonnegative solutions. Moreover, a nontrivial claim is that finite time blow up occurs iff the kinetic energy blows up. In the classical case (1.1), this feature can be seen for instance as a consequence of the propagation of moments proved by Lions and Perthame in [30]. In the relativistic case (1.3), no such property of propagation of moments is known, and the standard way to construct strong solutions is based on a control of the velocity support of $f$ (for discussions on this topic, see [1, 18, 21, 20, 22]). Theorem 10 in the relativistic case thus follows from the following estimate on the support in velocity of radially symmetric solutions to (1.3) which can be deduced from Glassey and Schaeffer [18: let

$$
P(t)=\sup \left\{|v|: \text { there exists }(x, s) \in \mathbb{R}^{3} \times[0, t] \text { with } f(s, x, v) \neq 0\right\} ;
$$

then one has: for all $t \in[0, T)$,

$$
P(t) \leq C\left(1+P(0)+\left|f_{0}\right|_{L^{\infty}}^{2}\left|f_{0}\right|_{L^{1}}^{3}\left|\sqrt{1+|v|^{2}} f(t)\right|_{L^{1}}\right) .
$$

Let us now recall that (1.1) and (1.3) are Hamiltonian systems, and the strong solutions of Theorem 1 satisfy the conservation of the $L^{q}$ norm:

$$
\forall t \in[0, T), \quad \forall q \in[1,+\infty], \quad|f(t)|_{L^{q}}=\left|f_{0}\right|_{L^{q}},
$$


and the Hamiltonian: let

$$
\begin{aligned}
& \text { (1.6) } \mathcal{H}(f(t)) \\
& =\left\{\begin{array}{l}
\frac{1}{2} \int_{\mathbb{R}^{2 N}}|v|^{2} f(t, x, v) d x d v-\frac{1}{2} \int_{\mathbb{R}^{N}}\left|E_{f}(t, x)\right|^{2} d x \text { for (1.1) with } N=3 \text { or } 4, \\
\int_{\mathbb{R}^{6}} \sqrt{1+|v|^{2}} f(t, x, v) d x d v-\frac{1}{2} \int_{\mathbb{R}^{3}}\left|E_{f}(t, x)\right|^{2} d x \text { for (1.3); }
\end{array}\right.
\end{aligned}
$$

then

$$
\forall t \in[0, T), \quad \mathcal{H}(f(t))=\mathcal{H}(f(0)) .
$$

In dimension $N=3$ for (1.1), the conservation of the $L^{1}, L^{p}$ norms (1.5) and the Hamiltonian (1.6) imply for $p>9 / 7$ a uniform bound on the kinetic energy thanks to the interpolation inequality

$$
\left|E_{f}\right|_{L^{2}}^{2} \leq C \|\left.\left. v\right|^{2} f\right|_{L^{1}} ^{1 / 2}|f|_{L^{1}}^{\frac{7 p-9}{6(p-1)}}|f|_{L^{p}}^{\frac{p}{3(p-1)}}
$$

Thus all classical solutions to (1.1) for $N=3$ are global in time from Theorem 1 .

The situation for the relativistic equation (1.3) or the four dimensional (1.1) is quite different. For $2<p<+\infty$, let the energy space be

$$
\mathcal{E}=\left\{f \geq 0 \text { with }|f|_{\mathcal{E}}=|f|_{L^{1}}+|f|_{L^{p}}+\left.\left.|| v\right|^{N-2} f\right|_{L^{1}}<+\infty\right\} .
$$

Then the interpolation inequality (1.8) becomes:

$$
\left|E_{f}\right|_{L^{2}}^{2} \leq C \|\left.\left. v\right|^{N-2} f\right|_{L^{1}}|f|_{L^{1}}^{\frac{2 p-N}{N(p-1)}}|f|_{L^{p}}^{\frac{p(N-2)}{N(p-1)}}
$$

and hence the strength of the kinetic and potential energies in the Hamiltonian (1.6) are now the same. The existence of finite time blow up solutions follows from the well known virial law: for (1.1) with $N=4$, it holds that

$$
\frac{1}{2} \frac{d^{2}}{d t^{2}} \int|x|^{2} f(t, x)=\mathcal{H}\left(f_{0}\right)
$$

which is in fact a consequence of the exact pseudo-conformal symmetry as exhibited in [26, and thus $\mathcal{H}\left(f_{0}\right)<0$ implies finite time blow up. For (1.3), the situation is a little more complicated but one can prove (see Glassey and Schaeffer [18]) that for radially symmetric initial data we have

$$
\int|x|^{2} f(t, x) \leq \mathcal{H}\left(f_{0}\right) t^{2}+c\left(f_{0}\right)(1+t)
$$

and thus the same conclusion holds. In short, nonpositive Hamiltonian classical solutions to (1.3) for $N=3$ or (1.1) for $N=4$ blow up in finite time.

In this paper, we study in a unified way the singularity formation for the VlasovPoisson system (1.1) in dimension $N=4$ and the relativistic Vlasov-Poisson system (1.3) in the physical case $N=3$ which are both critical type blow up problems.

1.2. About stable blow up dynamics for the $L^{2}$ critical NLS equation. The global structure of the problem of the singularity formation for (1.14) is reminiscent to the one for the $L^{2}$ critical nonlinear Schrödinger equation

$$
(N L S) \quad\left\{\begin{array}{l}
i u_{t}=-\Delta u-|u|^{\frac{4}{N}} u, \quad(t, x) \in[0, T) \times \mathbb{R}^{N}, \\
u(0, x)=u_{0}(x), \quad u_{0}: \mathbb{R}^{N} \rightarrow \mathbb{C},
\end{array}\right.
$$

both systems being nonlinear dispersive Hamiltonian systems with a critical growth nonlinearity. Recently, in the series of papers [32, 33, 34, 35, 36, 39, Merle and 
Raphaël obtained some sharp description results of the singularity formation for a specific class of initial data. An outcome of their work is in particular the fundamental role of the ground state stationary solutions in the description of the blow up dynamics. Indeed, let $R$ be the unique nonnegative radially symmetric solution in the energy space $H^{1}\left(\mathbb{R}^{N}\right)$ of

$$
\Delta R-R+R^{1+\frac{4}{N}}=0 .
$$

Then Weinstein 45] derived from the conservation of the Hamiltonian and the $L^{2}$ norm and the variational characterization of $R$ the following sharp criterion of global existence: let $u_{0} \in H^{1}$ with

$$
\left|u_{0}\right|_{L^{2}}<|R|_{L^{2}}
$$

the corresponding solution $u(t) \in H^{1}$ to (1.12) is global and bounded in $H^{1}$.

The analysis of Merle and Raphaël is focused on the study of small super critical blow up solutions. The following result which will further guide our intuition has been proved in [33, 39, 35, 13:

Theorem 2 (Existence of a stable log-log regime for (NLS)). Let $N=1,2,3,4,5$. There exist universal constants $\alpha^{*}, C^{*}>0$ such that the following holds true. Let an initial data

$$
u_{0} \in \mathcal{B}_{\alpha^{*}}=\left\{u_{0} \in H^{1} \text { with } \int R^{2}<\int\left|u_{0}\right|^{2}<\int R^{2}+\alpha^{*}\right\}
$$

with nonpositive Hamiltonian:

$$
E\left(u_{0}\right)=\frac{1}{2} \int\left|\nabla u_{0}\right|^{2}-\frac{1}{2+\frac{4}{N}} \int\left|u_{0}\right|^{2+\frac{4}{N}}<0 .
$$

Then the corresponding solution to (1.12) blows up in finite time $0<T<+\infty$ with a blow up speed

$$
|\nabla u(t)|_{L^{2}} \sim C^{*}\left(\frac{\log |\log (T-t)|}{T-t}\right)^{\frac{1}{2}} \text { as } t \rightarrow T .
$$

More generally, the set of initial data $u_{0} \in \mathcal{B}_{\alpha^{*}}$ such that the corresponding solution to (1.12) blows up in finite time with the blow up speed given by the log-log law (1.13) is open in $H^{1}$.

This theorem thus asserts the existence and the stability in the energy space of a singularity formation for which the blow up speed is given by the log-log law (1.13), which should be understood as a double-log correction to the more natural self-similar blow up speed. Moreover, the nonpositivity of the Hamiltonian is a sufficient condition of log-log blow up which corresponds to a strong classification result of the blow up dynamics.

1.3. Statement of the results. Following the intuition developed for the study of (NLS), in [26] we revisited the standard theory of orbital stability in the energy space of the ground states for (1.1) both in dimensions $N=3$ and $N=4$. Note that from the point of view of the singularity formation, the pertinent problem to study (1.3) and (1.1) for $N=4$ is to consider the unified homogeneous problem:

$$
\left\{\begin{array}{l}
\partial_{t} f+\frac{v}{|v|^{4-N}} \cdot \nabla_{x} f-E_{f} \cdot \nabla_{v} f=0, \quad(t, x, v) \in \mathbb{R}_{+} \times \mathbb{R}^{N} \times \mathbb{R}^{N}, \\
f(t=0, x, v)=f_{0}(x, v) \geq 0,
\end{array}\right.
$$


whose Hamiltonian is given by

$$
\mathcal{H}_{N}(f)=\frac{1}{N-2} \int_{\mathbb{R}^{2 N}}|v|^{N-2} f-\frac{1}{2} \int_{\mathbb{R}^{N}}\left|E_{f}\right|^{2}
$$

and for which the blow up problem is again critical according to (1.10). In dimension $N=3$, this model is known as the ultrarelativistic gravitational Vlasov-Poisson system, but we are not aware of any result concerning the Cauchy theory for this system. In [27] we generalized the construction of polytropic ground states to the critical homogeneous Vlasov-Poisson system (1.14). Given $p \in(2,+\infty)$, let $\phi_{Q}$ be the unique nontrivial radial solution to

$$
\frac{1}{r^{N-1}} \frac{d}{d r}\left(r^{N-1} \phi_{Q}^{\prime}\right)=\gamma_{N, p}\left(-1-\phi_{Q}\right)_{+}^{\frac{1}{p-1}+\frac{N}{N-2}}, \phi_{Q}(r) \rightarrow 0 \quad \text { as } r \rightarrow+\infty \text {, }
$$

where $g_{+}=\max (g, 0)$ and

$$
\gamma_{N, p}=(N-2)^{\frac{2}{N-2}} \sigma_{N} \int_{0}^{1} t^{\frac{2}{N-2}}(1-t)^{\frac{1}{p-1}} d t
$$

Note that the uniqueness of this object follows from the scaling invariance of (1.16). The corresponding spherically symmetric polytropic steady state solution to (1.14) is given by

$$
Q(x, v)=\left(-1-\frac{|v|^{N-2}}{N-2}-\phi_{Q}(x)\right)_{+}^{\frac{1}{p-1}} .
$$

We omit the $p, N$ dependence in $Q, \phi_{Q}, E_{Q}=\nabla_{x} \phi_{Q}$ to simplify the notation.

In [26] and following [45], we could derive the sharp criterion for the global existence of solutions to (1.14) in dimension $N=4$ on the basis of the variational characterization of $Q$ : let $f_{0}$ smooth and compactly supported with

$$
\left|f_{0}\right|_{L^{1}}\left|f_{0}\right|_{L^{p}}^{\frac{p}{p-2}}<|Q|_{L^{1}}|Q|_{L^{p}}^{\frac{p}{p-2}}
$$

then the corresponding classical solution to (1.14) for $N=4$ is global. This criterion is sharp from the existence of a pseudo-conformal symmetry: if $f(t, x, v)$ solves (1.1) for $N=4$, then given $T>0$, so does

$$
f\left(\frac{T t}{T-t}, \frac{T x}{T-t},(T-t) \frac{v}{T}+\frac{x}{T}\right) .
$$

Applying this to the ground state solution yields the explicit critical mass blow up solutions:

$$
S(t, x, v)=Q\left(\frac{T x}{T-t},(T-t) \frac{v}{T}+\frac{x}{T}\right) .
$$

The uniqueness of the critical mass blow up solution has moreover been proved in 28.

Our aim in this paper is to start the investigation of the singularity formation for both (1.14) for $N=4$ and (1.3) for $N=3$ for small super critical mass initial data.

From the mathematical point of view, the simplest problem is clearly (1.14) in dimension $N=4$ because of the existence of an explicit scaling symmetry: if $f(t, x)$ solves (1.14) for $N=4$, so does

$$
\left(\frac{\mu}{\lambda}\right)^{2} f\left(\frac{t}{\lambda \mu}, \frac{x}{\lambda}, \mu v\right), \quad \forall \lambda, \mu>0 .
$$


Our first result is the existence of a family of exact self-similar solutions to (1.14) in the energy space $\mathcal{E}$.

Proposition 1 (Exact self-similar solutions to (1.14) in $\mathcal{E}_{p}$ ). Let $N=3,4$ and $2<p<+\infty$. There exists a universal constant $b^{*}=b^{*}(p)>0$ such that for all $0<b<b^{*}$, there exists a compactly supported spherically symmetric stationary profile $Q_{b} \in \mathrm{C}^{0}\left(\mathbb{R}^{2 N}\right)$ such that

$$
f(t, x, v)=Q_{b}\left(\frac{x}{\lambda(t)}, \lambda(t) v\right) \text { with } \lambda(t)=[(N-2) b(T-t)]^{\frac{1}{N-2}}
$$

is an exact self-similar blow up solution to (1.14) in $\mathcal{E}$.

Comments on the result. 1. More general profiles: The self-similar profiles of Proposition 1 are constructed by a bifurcation technique from the polytropic steady states $Q$ in the sense that $Q_{b=0}=Q$. Let us recall that any smooth convex functional $j$ generates in a natural way a ground state stationary solution $Q(j)$ to (1.14) (see [2], 23], 26]), the polytropic steady state $Q$ corresponding to the choice $j(f)=f^{p}$. However, in this frame, uniqueness for the corresponding variational problems is not known in most instances, unless the results by Lieb and Yau [29] (see also Schaeffer [43]) apply. In this paper we have chosen to work only with the polytropic steady states because uniqueness is then straightforward in this case thanks to the scaling invariance of (1.16). Nevertheless, the constructive method of section 2 would clearly adapt to obtain self-similar profiles by bifurcation from the ground states $Q(j)$.

2. About Jean's theorem: The stationary profile $Q_{b}$ will be constructed as a ground state solution to the stationary self-similar equation

$$
\frac{v}{|v|^{4-N}} \cdot \nabla_{x} g-E_{g} \cdot \nabla_{v} g+b\left(x \cdot \nabla_{x} g-v \cdot \nabla_{v} g\right)=0 .
$$

In the case $b=0$ and for $N=4$, this reduces to the stationary equation for (1.14):

$$
v \cdot \nabla_{x} g-E_{g} \cdot \nabla_{v} g=0
$$

whose smooth enough solutions are known to satisfy the so-called Jean's theorem: from Batt, Faltenbacher and Horst 2, all spherically symmetric solutions $g$ to (1.22) are functions of their microscopic energy:

$$
g(x, v)=F\left(\frac{|v|^{2}}{2}+\phi_{g}(x)\right) .
$$

On the contrary, for $b \neq 0$, it can be seen that any solution $g$ to (1.21) of the form

$$
g(x, v)=F\left(\frac{|v|^{N-2}}{N-2}+b x \cdot v+\phi_{g}(x)\right)
$$

for a natural class of $F$ (see Dolbeault [12]) is not in the energy space $\mathcal{E}$. Now the Jean's theorem turns out to be false for (1.21) as soon as $b \neq 0$ and indeed the self-similar profiles $Q_{b}$ of Proposition 1 are not functions of their microscopic energy.

3. A comparison with the $L^{2}$ critical $N L S$ equation: In dimension $N=4$, the self-similar equation (1.21) is equivalent up to a change of variables to

$$
v \cdot \nabla_{x} P_{b}-\left(E_{P_{b}}-b^{2} x\right) \cdot \nabla_{v} P_{b}=0
$$


and thus the influence of $b \neq 0$ corresponds to the presence of a global defocusing harmonic potential $-\frac{b^{2}}{2}|x|^{2}$. This is exactly the same as for the $L^{2}$ critical NLS equation (1.12) for which the self-similar equation can be reduced to

$$
\Delta P_{b}-P_{b}+\frac{b^{2}|x|^{2}}{4} P_{b}-P_{b}\left|P_{b}\right|^{\frac{4}{N}}=0
$$

see 33 . Knowing both problems, if one tries to build a solution to the self-similar equation by bifurcation from the ground state solution at $b=0$, a major difference occurs between (1.24) and (1.23). For the (NLS) problem, the ground state $Q$ never vanishes and has exponential decay in space as $|x| \rightarrow+\infty$. This means that for $|x| \geq \frac{2}{|b|}$, the harmonic potential in (1.24) becomes dominant and induces an oscillation of the solution. Nevertheless, this tunneling effect appears at very large $|x|$ where $Q$ decays exponentially, and we are then left with an exponentially small tail at infinity. As a conclusion, there are no solutions to the self-similar equation (1.24) in the energy space, but one can construct approximate solutions with an exponentially small in $b$ error; see [33. This exponential smallness is at the heart of the log-log correction to the blow up speed according to the log-log law (1.13); see [35. On the contrary, for (1.23), the ground state is compactly supported and our main observation here is to see that despite the fact that the nonlinearity is nonlocal, one can build compactly supported solutions to (1.23) for which the harmonic potential is thus always a small perturbation. This is a major qualitative difference between (NLS) and (VP) regarding the blow up dynamics.

The existence of exact self-similar blow up solutions for (1.14) relies on the study of the stationary self-similar equation (1.21) which is a nonlinear elliptic problem. This strategy would fail to build exact self-similar solutions to the relativistic equation (1.3) which does not have an exact scaling symmetry. Our main claim in this paper is now that the self-similar profiles of Proposition 1 are dynamically stable. Moreover, they generate blow up dynamics where (1.14) in dimension $N=3$ is indeed a good approximation to the relativistic equation (1.3), and this allows us to derive the existence of stable self-similar blow up solutions to (1.3). Our main result is the following:

Theorem 3 (Stable self-similar blow up dynamics). Let $N=3,4$ and $2<p<$ $+\infty$. There exists a universal constant $b^{*}=b^{*}(N, p)>0$ such that the following holds true. Let $0<b_{0}<b^{*}$ and $Q_{b_{0}}$ be the self-similar profiles to (1.14) given by Proposition 1 then there exists $\lambda\left(b_{0}\right)>0$ such that the following holds true. Let $0<\lambda_{0}<\lambda\left(b_{0}\right)$. There exists $\alpha\left(\lambda_{0}\right)>0$ such that for all initial data $f_{0} \in \mathcal{C}_{0, \text { rad }}^{1}$ with

$$
\left|Q_{b_{0}}\left(\frac{x}{\lambda_{0}}, \lambda_{0} v\right)-f_{0}\right|_{\varepsilon}<\alpha\left(\lambda_{0}\right),
$$

the corresponding classical solution $f(t)$ to respectively (1.3) for $N=3$ or (1.14) for $N=4$ blows up in finite time $0<T<+\infty$ according to the following self-similar blow up dynamics. Let

$$
\lambda_{f}^{N-2}(t)=\frac{\left.\left.|| v\right|^{N-2} Q\right|_{L^{1}}}{\left.\left.|| v\right|^{N-2} f(t)\right|_{L^{1}}}
$$

then $f(t)$ admits a decomposition

$$
f(t, x, v)=Q\left(\frac{x}{\lambda_{f}(t)}, \lambda_{f}(t) v\right)+\tilde{f}(t, x, v)
$$


where the singular part forms a Dirac mass in $L^{1}$ at the speed

$$
\frac{b_{0}(T-t)}{4} \leq \lambda_{f}^{N-2}(t) \leq 4 b_{0}(T-t),
$$

and $\tilde{f}$ is globally $L^{1}$ small and remains smooth outside the origin in the sense that

$$
\forall R>0, \quad(1+|v|) \tilde{f} \rightarrow(1+|v|) f^{*} \quad \text { in } \quad L^{1}(|x|>R) \quad \text { as } t \rightarrow T .
$$

Comments on the result. 1 . On the sign of the Hamiltonian: Theorem 3 thus asserts the existence and the stability under radially symmetric perturbations of self-similar solutions for both (1.3) and (1.14) for $N=4$. Note that our need to pick compactly supported initial data is based only on the Cauchy theory which is not satisfactory enough to work only in the natural energy space $\mathcal{E}$. Note also that, at least in the case (1.1) with $N=4$, the sign of the Hamiltonian is not prescribed around $Q_{b_{0}}$ which has zero Hamiltonian, and thus we obtain finite time blow up solutions with either sign of the energy.

2. On the asymptotic behavior of the system at blow up time: A complete description of the blow up dynamics would require the precise understanding of the behavior of $\tilde{f}$ as $t \rightarrow T$. Let us observe that - on the contrary to what happens for (NLS), see [36]- the regular part $\tilde{f}$ may form a small Dirac mass at the origin. This is due to the presence of self-similar solutions with a blow up profile different from $Q$ in any neighborhood of $Q$. A simpler question is the existence in rescaled variables of a strong limit of the solution as $t \rightarrow T$. Equivalently, does the solution select an asymptotic blow up profile at blow up time? In rescaled variables, the solution disperses to zero away from the soliton core in time averaging sense as a consequence of the dispersive control (3.23). However, the understanding of the behavior of the rescaled solution near the soliton core would require going further than our so far poor understanding of the dispersive dynamics of the system on the soliton core. Hence an oscillation phenomenon is a priori possible; see section 3.2. Note that this issue in the subcritical setting of the three dimensional gravitational Vlasov-Poisson is unclear as well. However, some averaged quantities do have a strong limit as $t \rightarrow T$ as a consequence of (1.28). Indeed, we have in particular:

$$
\rho_{f}(t)=\int_{v \in \mathbb{R}^{N}} f(t, x, v) d v \text { has a strong limit in the measure sense as } t \rightarrow T .
$$

To wit, let us focus on (1.14) for $N=4$ first and pick a smooth compactly supported $\psi$. We compute from integration by parts and the estimate on the blow up speed (1.28):

$$
\left|\frac{d}{d t} \int_{\mathbb{R}^{N}} \psi(x) \rho_{f}(x) d x\right|=\left|\int_{\mathbb{R}^{2 N}} v \cdot \nabla \psi f d x d v\right| \leq\left.\left. C_{\psi}|| v\right|^{2} f\right|_{L^{1}} ^{\frac{1}{2}}|f|_{L^{1}}^{\frac{1}{2}} \leq \frac{C\left(\psi, b_{0}, f\right)}{\sqrt{T-t}}
$$

from which

$$
\int_{0}^{T}\left|\frac{d}{d t} \int_{\mathbb{R}^{N}} \psi(x) \rho_{f}(x) d x\right| d t<+\infty
$$

and the claim follows. The case of (1.3) is even simpler.

3. Regularity outside the blow up point: The gain of one momentum outside the singularity (1.29) should be compared to the gain of half a derivative for (NLS); see [40]. Note that this gain is, remarkably enough, optimal in the energy space for (1.3). 
4. Stable blow up dynamics: The fact that the relativistic term $\frac{v}{\sqrt{1+|v|^{2}}}$ breaks down the exact scaling symmetry for (1.3) classically implies the existence and stability of globally defined ground state solutions with subrcitical mass; see also 24. Together with Theorem 3. this shows that there exist at least two stable dynamics for (1.3): a subcritical global dynamic near well chosen ground states, and the self-similar blow up dynamics described by Theorem 3 . A similar situation is encountered for the $L^{2}$ critical (NLS) on a bounded domain with Dirichlet boundary condition; see Planchon and Raphael [38. Another astrophysical model which displays a similar structure is the pseudo-relativistic Hartree model for boson stars; see the recent works by Frohlich, Jonsson and Lenzman [16], Frohlich and Lenzman [15].

5. Other blow up dynamics: Let us observe that the critical mass blow up solution (1.20) to (1.1) for $N=4$ does not blow up with the self-similar speed (1.28) but with

$$
\left.\left.|| v\right|^{2} S_{p}(t)\right|_{L^{1}} \sim \frac{C}{(T-t)^{2}} .
$$

Moreover, any neighborhood of $Q$ in $\mathcal{E}$ contains an initial data $f_{0} \in \mathcal{C}_{0, \text { rad }}^{1}$ such that the corresponding solution to (1.1) in dimension $N=4$ blows up in finite time $0<T<+\infty$ with the excited blow up rate (1.30): it suffices to apply the pseudoconformal symmetry (1.19) to a ground state solution obtained from some convex functional $j$ and close to $Q$. Another strategy to build such an object would be to consider the solution to (1.1) with initial data at time $t_{0} \gg 1: f\left(t_{0}\right)=Q+\tilde{f}\left(t_{0}\right)$ with

$\tilde{f}_{0}$ radially symmetric and $\operatorname{Supp}\left(\tilde{f}\left(t_{0}\right)\right) \subset\{R \leq|x| \leq 2 R, 1 \leq|v| \leq 2, x \cdot v \geq R\}$.

Integrating (1.1) thanks to the explicit characteristics of the transport by $E_{Q}$ and using the decay of $E_{Q}$ given by Lemma 2 , it is easily seen that for $t_{0}, R$ large enough, it holds that $\forall t \geq t_{0}$,

$$
\begin{aligned}
& f(t)=Q+\tilde{f}(t) \\
& \quad \text { with } \operatorname{Supp}(\tilde{f}(t)) \subset\left\{\frac{R+t}{16} \leq|x| \leq 4(R+t), \frac{1}{2} \leq|v| \leq 4, x \cdot v \geq \frac{R+t}{4}\right\} .
\end{aligned}
$$

This construction, which is also valid for (1.1) and $N=3$, thus ensures the existence of nontrivial global solutions which disperse asymptotically to the ground state:

$$
f(t) \rightarrow Q \text { in } L_{l o c}^{1} \text { as } t \rightarrow+\infty
$$

We then apply in dimension $N=4$ the pseudo-conformal symmetry (1.19) to this object and get a finite time blow up solution blowing up with the excited blow up speed (1.30) and blow up profile exactly $Q$. Note that this kind of result for (NLS) or (KdV) type of problems is much more difficult to reach; see for example Bourgain and Wang [5, Krieger and Schlag [25] or Cote 7].

If we now compare Theorem 3 with Theorem 2 for (NLS), one of the major open problems after this work is the following classification problem: let a smooth initial data with small super critical mass $|Q|_{L^{1}}|Q|_{L^{p}}^{\frac{p}{p-2}}<\left|f_{0}\right|_{L^{1}}\left|f_{0}\right|_{L^{p}}^{\frac{p}{p-2}}<|Q|_{L^{1}}|Q|_{L^{p}}^{\frac{p}{p-2}}+\alpha^{*}$, $0<\alpha^{*} \ll 1$, and nonpositive Hamiltonian $\mathcal{H}\left(f_{0}\right)<0$ so that the corresponding solution to (1.1) in dimension $N=4$ blows up in finite time $0<T<+\infty$ from the 
virial law (1.11), is the blow up rate given by the stable self-similar law (1.28) or is the excited blow up rate (1.30) -or others- possible?

1.4. Strategy of the proof. Let us give a brief overview of the proof of Theorem 3. The general strategy relies on a similar description of the singularity formation as in the works by Merle and Raphaël on (NLS) 32, 33, 39, 35, and on a bootstrap argument in the spirit of the works by Planchon and Raphaël [38, and Raphaël [40. Given $b_{0}>0$, we pick a smooth radially symmetric initial data $f_{0} \geq 0$ of the form

$$
f_{0}(x)=\left(Q_{b_{0}}+\varepsilon_{0}\right)\left(\frac{x}{\lambda_{0}}, \lambda_{0} v\right)
$$

for some small enough $\lambda_{0}>0$ and $\left|\varepsilon_{0}\right| \varepsilon \ll 1$. We let $f \in \mathcal{C}^{1}\left([0, T), \mathcal{C}_{0, \text { rad }}^{0}\right), 0<T \leq$ $+\infty$, the corresponding maximal solution to (1.3) for $N=3$ or (1.14) for $N=4$ given by Theorem 1. The space-time regularity of $f$ ensures that the structure (1.31) remains for a short time and we have a geometrical decomposition

$$
f(t, x)=\left(Q_{b(t)}+\varepsilon\right)\left(\frac{x}{\lambda(t)}, \lambda(t) v\right)
$$

with $|\varepsilon(t)|_{\mathcal{E}} \ll 1$. From modulation theory, the uniqueness of the geometrical parameters $\lambda(t), b(t)$ and the excess of mass $\varepsilon(t)$ is ensured by imposing some suitable orthogonality conditions on $\varepsilon(t)$; see Proposition 4 . Our strategy is the following: assuming at time $t=0$ suitable estimates on $\lambda_{0}$ and $\varepsilon_{0}$ of the form

$$
\lambda_{0}+\left|\varepsilon_{0}\right|_{\mathcal{\varepsilon}} \ll b_{0},
$$

we aim at proving that this configuration is stable along the flow of (1.3) or (1.14). In particular, we prove using a bootstrap argument that the geometrical decomposition (1.32) holds for all $\in[0, T)$ with an estimate:

$$
\forall t \in[0, T), \quad \frac{b_{0}}{2} \leq b(t) \leq 2 b_{0} \text { and }|\varepsilon(t)|_{\varepsilon} \ll b(t) .
$$

Assume (1.33); then the modulation equation for the scaling parameter $\lambda$, which is a consequence of our choice of orthogonality conditions for $\varepsilon(t)$, and (1.33) implies

$$
\left|\frac{d}{d t} \lambda^{N-2}+b(t)\right| \leq C|\varepsilon(t)|_{\varepsilon} \ll b(t) \text { and thus } \frac{b_{0}}{2} \leq-\frac{d}{d t} \lambda^{N-2} \leq 2 b_{0} .
$$

Observe now from (1.32) and the smallness of $|\varepsilon(t)|_{\varepsilon}$ that

$$
\lambda^{N-2}(t) \sim \frac{\|\left.\left. v\right|^{N-2} Q\right|_{L^{1}}}{\|\left.\left. v\right|^{N-2} f(t)\right|_{L^{1}}}=\lambda_{f}^{N-2}(t)
$$

so that the time integration of (1.34) implies

$$
T<+\infty \text { and } \frac{b_{0}}{4}(T-t) \leq \lambda_{f}^{N-2}(t) \leq 4 b_{0}(T-t),
$$

and (1.28) follows.

The heart of the analysis is thus the proof of the estimate (1.33) which means that the infinite dimensional dynamic may be reduced somehow to the control of the one dimensional dynamic governing the geometrical parameter $b$. Our main claim is the existence of a local virial type dispersive estimate inherited from the 
global virial law (1.11) and our specific choice of orthogonality conditions for $\varepsilon(t)$ which implies the existence of a Lyapounov function in time which is essentially

$$
\frac{d b}{d t} \geq \frac{1}{\lambda^{N-2}} \int_{|x||v| \geq A}|v|^{N-2} \varepsilon \geq 0 .
$$

Note that the choice of the constant $A$ is related to the structure of the characteristics of $Q$ and the dispersive structure of the linearized operator close to $Q$ as exhibited in 27. Such an estimate was derived for the first time for (NLS) in [32] as a consequence in particular of positivity properties of suitable quadratic forms involving the ground state $Q$, and which could not be fully proved analytically but were checked numerically; see 13 . Here on the contrary, we exhibit for (1.3) and (1.14) a complete dynamical decoupling between the ground state part of the solution and the excess of mass $\varepsilon(t)$ at the level of the local virial identity which implies (1.35); see the proof of Lemma 4. Note that the radial assumption is used here in a crucial way to get some decay on the gravitational field and ensure the corresponding decoupling of the interactions in the Morawetz type identity leading to (1.35). Then (1.35) implies that $b$ is nondecreasing and the lower bound on $b$ in (1.33) follows.

The upper bound on $b(t)$ and $|\varepsilon(t)|_{\mathcal{E}}$ in (1.33) is now a consequence of the conservation of the Hamiltonian and the $L^{1}, L^{p}$ norms. Here we use the complete understanding of the coercivity properties of the linearized operator close to the ground state $Q$ as obtained in 27 and which is a consequence of the sharp understanding of the variational characterization of the ground state as derived in [26].

It remains to prove the regularity (1.29) outside the origin which is a consequence of the virial dispersive estimate (1.35). Indeed, let

$$
\tilde{f}(t, x, v)=\varepsilon\left(\frac{x}{\lambda(t)}, \lambda(t) v\right)
$$

then (1.35) implies

$$
\forall R>0, \quad \int_{0}^{T} \int_{|x| \geq R}|v|^{N-2} \tilde{f}(t, x, v) d x d v<C(R) .
$$

We then write the equation for $\tilde{f}$ outside the origin in the Duhamel form and observe that the space-time gain of regularity (1.36) together with the uniform $L^{\infty}$ bound on the gravitational field away from zero from our radial assumption imply (1.29).

This paper is organized as follows. In section 2, we construct the self-similar profiles $Q_{b}$ solution to (1.21) using nonlinear elliptic techniques by bifurcation from the ground state $Q$. We then introduce a geometrical decomposition (1.32) for the solutions close in some sense to $Q$. In section 3 , we give an explicit description of the set of initial data leading to the blow up solutions of Theorem 3 and set up the bootstrap argument. We then derive the two fundamental estimates at the heart of our analysis: the virial type dispersive estimate, section 3.2 , and the pointwise control of $|\varepsilon|_{\varepsilon}$ induced by the linearization of the conservation laws, section 3.3. This allows us to conclude the proof of the bootstrap Lemma 3, section 3.4. We then conclude in section 3.5 the proof of Theorem 3 , 


\section{Geometrical DeComposition of the SOlution}

This section is devoted to the introduction of a geometrical decomposition of a suitable class of solutions for (1.14) and (1.3). The first step is the construction of a one parameter family of self-similar profiles $Q_{b}$ which are exact compactly supported solutions to the self-similar equation (1.21) and are obtained by bifurcation from the ground state polytropic solutions $Q$ at $b=0$. A key step here is the complete understanding of the linear operator close to the $Q$ which is a consequence of the analysis in [27] and allows one to view the $Q_{b}$ as a smooth in $b$ deformation of $Q$. We then introduce a geometrical decomposition of the solutions as long as it remains close to the two dimensional manifold

$$
\mathcal{M}=\left\{Q_{b}\left(\frac{x}{\lambda}, \lambda v\right), \quad(b, \lambda) \in(-1,1) \times \mathbb{R}_{+}^{*}\right\}
$$

where the parameter $b$ corresponds to the self-similar degeneracy of the problem.

2.1. Exact self-similar solutions to (1.14). This subsection is devoted to the construction of exact solutions to the self-similar equation (1.14) both in dimension $N=3$ and $N=4$. The proof of Proposition 1 relies on standard nonlinear elliptic techniques. As we pointed out in comment 3 after Proposition 11, the fact that $Q$ is compactly supported implies that the corrective harmonic potential in the self-similar equation (1.21) can always be treated as a small perturbation.

We begin with constructing the self-similar potentials. Let $N=3$ or $4,2<p<$ $+\infty, \phi_{Q}$ given by (1.16) be the field of the polytrope $Q$ and $r_{Q} \in(0,+\infty)$ be the unique solution to

$$
\phi_{Q}\left(r_{Q}\right)=-\frac{1}{2}
$$

We fix once and for all a smooth radially symmetric cut-off function

$$
\chi_{p}(x)=\left\{\begin{array}{l}
1 \text { for }|x| \leq 10 r_{Q}, \\
0 \text { for }|x| \geq 20 r_{Q}
\end{array}\right.
$$

and claim:

Proposition 2 (Construction of the self-similar potentials). Let $N=3,4$. There exist universal constants $b^{*}=b^{*}(p)>0, \delta^{*}=\delta^{*}(p)>0$ such that for all $|b|<b^{*}$, there exists a unique radially symmetric solution $\phi_{b} \in \mathrm{C}^{2}\left(\mathbb{R}^{N}\right)$ to

$$
\left\{\begin{array}{l}
\frac{1}{r^{N-1}} \frac{d}{d r}\left(r^{N-1} \phi_{b}^{\prime}\right)=\int_{v \in \mathbb{R}^{N}}\left(-\frac{|v|^{N-2}}{N-2}-1-b \chi_{p}(x) x \cdot v-\phi_{b}\right)_{+}^{\frac{1}{p-1}} d v \\
\left|\phi_{b}(0)-\phi_{Q}(0)\right|<\delta^{*} \\
\phi_{b}(r) \rightarrow 0 \text { as } r \rightarrow+\infty
\end{array}\right.
$$

Moreover, we have the following properties:

(i) Localization of $\phi_{b}$ :

$$
\forall r \geq 2 r_{Q}, \quad-\frac{2}{3}<\phi_{b}(r) \leq 0 ;
$$

(ii) Smoothness of the flow: the application

$$
b \rightarrow \phi_{b} \quad \text { is } \quad \mathrm{C}^{1}\left(\left(-b^{*}, b^{*}\right), \mathrm{C}^{2}\left(\mathbb{R}^{N}\right) \cap \dot{H}^{1}\right),
$$


and

$$
\phi_{b}(b=0)=\phi_{Q} \quad \text { and } \quad \frac{\partial \phi_{b}}{\partial b}(b=0)=0 .
$$

Proof of Proposition 2. Proposition 2 follows from standard variational techniques. The uniqueness of $\phi_{b}$ relies however on the knowledge of the spectral structure of the linear operator of (2.3) close to $\phi_{Q}$ which is a nontrivial consequence of the variational characterization of $\phi_{Q}$; see Lemma 1, which has been proved in 27. Here we will walk in the steps of [33].

Step 1. Variational construction of $\tilde{Q}_{\tilde{b}}$.

Let $N=3,4, p \in(2,+\infty)$ and

$$
\mathcal{E}_{r}=\{f \in \mathcal{E} \text { with radial symmetry }\}
$$

where the energy space $\mathcal{E}$ is given by (1.9). Let us recall that radial symmetry means that the function depends only on $(|x|,|v|, x \cdot v)$. Let $\chi_{p}$ be the smooth cut-off function (2.2). Let $\tilde{b} \in\left(-b^{*}, b^{*}\right)$ for some $b^{*}>0$ small enough to be chosen later. Let

$$
T(f)=\frac{1}{N-2} \int_{\mathbb{R}^{2 N}}|v|^{N-2} f+\int_{\mathbb{R}^{2 N}} f+\tilde{b} \int_{\mathbb{R}^{2 N}} \chi_{p}(x)(x \cdot v) f+\frac{1}{p} \int_{\mathbb{R}^{2 N}} f^{p}
$$

and consider the minimization problem

$$
T^{*}(\tilde{b})=\inf _{f \in \mathcal{E}_{r}, \quad\left|E_{f}\right|_{L^{2}}=\left|E_{Q}\right|_{L^{2}}} T(f) .
$$

First observe from (2.2) that

$$
\forall(x, v), \quad \frac{|v|^{N-2}}{N-2}+1+\tilde{b} \chi_{p}(x) x \cdot v \geq \frac{1}{4}\left(|v|^{N-2}+1\right)
$$

for $|\tilde{b}|<b^{*}$ small enough and thus

$$
T(f) \geq \frac{1}{4} \int_{\mathbb{R}^{2 N}}\left(|v|^{N-2}+1\right) f+\frac{1}{p} \int_{\mathbb{R}^{2 N}} f^{p} \text { and } T^{*}(\tilde{b}) \geq 0 .
$$

Let $f_{n} \in \mathcal{E}_{r}$ be a minimizing sequence with spherical symmetry; then $\left|f_{n}\right| \varepsilon_{r} \leq C$ from (2.8) and

$$
f_{n} \rightarrow \tilde{Q}_{\tilde{b}} \text { in } \mathcal{E}_{r} \text { as } n \rightarrow+\infty
$$

up to a subsequence. Moreover, the spherical symmetry of $f_{n}$ implies that $\rho_{f_{n}}$ is spherically symmetric so that from a standard compactness result (see Appendix $\mathrm{C}$ in [26])

$$
E_{f_{n}} \rightarrow E_{\tilde{Q}_{\tilde{b}}} \text { in } L^{2} \text { as } n \rightarrow+\infty .
$$

From (2.7) and Fatou's Lemma, we conclude that $\tilde{Q}_{\tilde{b}}$ attains the infimum

$$
T\left(\tilde{Q}_{\tilde{b}}\right)=T^{*}(\tilde{b})>0, \quad\left|E_{\tilde{Q}_{\tilde{b}}}\right|_{L^{2}}=\left|E_{Q}\right|_{L^{2}}
$$

Let us observe that

$$
\frac{1}{C}<\left|\tilde{Q}_{\tilde{b}}\right|_{\mathcal{E}}<C
$$

for some universal constant $C>0$ uniformly for $|\tilde{b}|<b^{*}$. Indeed, the upper bound is a consequence of $T\left(\tilde{Q}_{\tilde{b}}\right)=T^{*}(\tilde{b})<C$ uniformly for $|\tilde{b}|<b^{*}$ small enough and the lower bound on $T(f)$ given by (2.8). The lower bound follows from the constraint $\left|E_{\tilde{Q}_{\tilde{b}}}\right|_{L^{2}}=\left|E_{Q}\right|_{L^{2}}$ and the Gagliardo-Nirenberg interpolation inequality (1.10). 
From standard Lagrange multiplier theory (see for example 23] for further details) there exists a Lagrange multiplier $\nu(\tilde{b})$ such that

$$
\frac{|v|^{N-2}}{N-2}+1+\tilde{b} \chi_{p}(x) x \cdot v+\tilde{Q}_{\tilde{b}}^{p-1}=-\nu(\tilde{b}) \phi_{\tilde{Q}_{\tilde{b}}} \text { a.e. on } \operatorname{Supp}\left(\tilde{Q}_{\tilde{b}}\right) .
$$

Taking the inner product of (2.11) with $\tilde{Q}_{\tilde{b}}$ and using (2.9), we conclude:

$$
\nu(\tilde{b})\left|E_{\tilde{Q}_{\tilde{b}}}\right|_{L^{2}}^{2}=\nu(\tilde{b})\left|E_{Q}\right|_{L^{2}}^{2}=T^{*}(\tilde{b})+\left(1-\frac{1}{p}\right) \int_{\mathbb{R}^{2 N}} \tilde{Q}_{\tilde{b}}^{p},
$$

and thus (2.10) implies

$$
0<\nu_{1}<\nu(\tilde{b})<\nu_{2}
$$

for some universal constants $\nu_{1}, \nu_{2}>0$ uniformly for $|\tilde{b}|<b^{*}$. Integrating (2.11) in $v$, we get the equation for the potential $\tilde{\phi}_{\tilde{b}}=\phi_{\tilde{Q}_{\tilde{b}}}$ :

$$
\Delta \tilde{\phi}_{\tilde{b}}=\int_{v \in \mathbb{R}^{N}}\left(-\frac{|v|^{N-2}}{N-2}-1-b \chi_{p}(x) x \cdot v-\nu(\tilde{b}) \tilde{\phi}_{\tilde{b}}\right)_{+}^{\frac{1}{p-1}} d v .
$$

Moreover, let $q=\frac{2 N p-2 p-N}{N p-2}, r=\frac{N q}{N-2 q}$; then the standard interpolation estimates

$$
\begin{gathered}
\left|\rho_{f}\right|_{L^{q}} \leq\left.\left. C|| v\right|^{N-2} f\right|_{L^{1}} ^{\frac{N(p-1)}{2 N-2 p-N}}|f|_{L^{p}}^{\frac{p(N-2)}{2 N p-2 p-N}}, \\
\left|\phi_{f}\right|_{L^{r}}=\left|\frac{1}{(N-2) \sigma_{N}|x|^{N-2}} \star \rho_{f}\right|_{L^{r}} \leq C\left|\rho_{f}\right|_{L^{q}}
\end{gathered}
$$

imply together with (2.10) that

$$
\left|\tilde{\phi}_{\tilde{b}}\right|_{L^{r}} \leq C
$$

uniformly for $|\tilde{b}|<b^{*}$.

Step 2. Uniform estimates on $\tilde{\phi}_{\tilde{b}}$ in $\mathcal{C}^{2}$.

We now claim from (2.13), (2.14) and standard bootstrap arguments that there exists $0<\alpha<1$ such that

$$
\left|\tilde{\phi}_{\tilde{b}}\right|_{e^{2, \alpha}} \leq C \text { and } \tilde{\phi}_{\tilde{b}}(r) \rightarrow 0 \text { as } r \rightarrow \infty \text { uniformly for }|\tilde{b}|<b^{*} .
$$

Proof of (2.15). First observe that the nonlinearity in (2.13) is explicit: let

$$
G(a, b)(X)=\int_{v \in \mathbb{R}^{N}}\left(-\frac{|v|^{N-2}}{N-2}-b X \cdot v+a\right)_{+}^{\frac{1}{p-1}} d v ;
$$

then an explicit computation gives for $|b X|<\frac{1}{2}$ :

$$
\begin{aligned}
& G(a, b)=2 \sigma_{4}\left(a+\frac{|b X|^{2}}{2}\right)_{+}^{\frac{1}{p-1}+2} \int_{0}^{1} u(1-u)^{\frac{1}{p-1}} d u \text { for } N=4, \\
& G(a, b)=\sigma_{3} \frac{a_{+}^{\frac{1}{p-1}}+3}{\left(1-|b X|^{2}\right)^{2}} \int_{0}^{1} u^{2}(1-u)^{\frac{1}{p-1}} d u \text { for } N=3 .
\end{aligned}
$$

We now rewrite (2.13):

$$
\tilde{\rho}_{\tilde{b}}=\Delta \tilde{\phi}_{\tilde{b}}=G\left(-1-\nu(\tilde{b}) \tilde{\phi}_{\tilde{b}}(x), \tilde{b}\right)\left(x \chi_{p}(x)\right)
$$


and observe that $\left|\tilde{b} x \chi_{p}(x)\right| \leq \frac{1}{2}$ for $|\tilde{b}|<b^{*}$ small enough. Moreover,

$$
G\left(-1-\nu(\tilde{b}) \tilde{\phi}_{\tilde{b}}, \tilde{b}\right) \leq C\left(-\frac{1}{2}-\nu(\tilde{b}) \tilde{\phi}_{\tilde{b}}\right)_{+}^{\frac{1}{p-1}+\frac{N}{N-2}} \leq C\left|\tilde{\phi}_{\tilde{b}}\right|^{\frac{1}{p-1}+\frac{N}{N-2}} .
$$

We conclude that if $\tilde{\rho}_{\tilde{b}} \in L^{q_{k}}$, then $\tilde{\phi}_{\tilde{b}}=\frac{1}{(N-2) \sigma_{N}|x|^{N-2}} \star \tilde{\rho}_{\tilde{b}} \in L^{r_{k}}$ with $r_{k}=\frac{N q_{k}}{N-2 q_{k}}$ from Hardy, Littlewood, Sobolev so that $\tilde{\rho}_{\tilde{b}} \in L^{q_{k+1}}$ with

$$
q_{k+1}\left(\frac{1}{p-1}+\frac{N}{N-2}\right)=r_{k}, \text { i.e. } q_{k+1}=\frac{(p-1)(N-2)}{N p-2} \frac{N q_{k}}{N-2 q_{k}} .
$$

A simple analysis of the sequence $q_{k}$ with $q_{0}=\frac{2 N p-2 p-N}{N p-2}$ shows that $p>2 \geq \frac{N}{2}$ implies that there exists $k_{0}=k(N, p)$ such that

$$
q_{k_{0}}>\frac{N}{2} \text { and thus }\left|\tilde{\rho}_{\tilde{b}}\right|_{L^{q_{k_{0}}}} \leq C
$$

uniformly for $|\tilde{b}|<b^{*}$. From Sobolev injections, this implies $\tilde{\phi}_{\tilde{b}} \in \mathcal{C}_{l o c}^{0, \alpha\left(k_{0}\right)}$ for some $0<\alpha\left(k_{0}\right)<1$ and thus standard bootstrap arguments imply

$$
\left|\tilde{\phi}_{\tilde{b}}\right|_{\mathcal{C}_{l o c}^{2, \alpha\left(k_{0}\right)}} \leq C \text { uniformly in }|\tilde{b}|<b^{*} .
$$

We may now solve the Poisson equation in radial coordinates:

$$
0 \leq-\tilde{\phi}_{\tilde{b}}(r)=\int_{r}^{+\infty} \frac{d u}{u^{N-1}} \int_{0}^{u} w^{N-1} \tilde{\rho}_{\tilde{b}}(w) d w \leq \frac{\left|\tilde{\rho}_{\tilde{b}}\right|_{L^{1}}}{(N-2) \sigma_{N} r^{N-2}} .
$$

We conclude from (2.10) that $\tilde{\phi}_{\tilde{b}}(r) \rightarrow 0$ as $r \rightarrow+\infty$ uniformly for $|\tilde{b}|<b^{*}$. From (2.12) and (2.13), this implies that the support of $\Delta \tilde{\phi}_{\tilde{b}}$ lies in a fixed compact for $|\tilde{b}|<b^{*}$ and thus there exists $r_{0}$ independent of $\tilde{b}$ such that

$$
\forall r \geq r_{0}, \quad \tilde{\phi}_{\tilde{b}}(r)=-\frac{\left|\tilde{\rho}_{\tilde{b}}\right|_{L^{1}}}{(N-2) \sigma_{N} r^{N-2}} .
$$

Finally, (2.15) follows from (2.10), (2.18) and (2.19).

Step 3. Strong convergence to $\phi_{Q}$ as $\tilde{b} \rightarrow 0$.

We now let $\tilde{b} \rightarrow 0$. From (2.12), (2.15) and the $\mathcal{C}^{1}$ regularity of $G$ from (2.16), (2.17) and $\left|\tilde{b} x \chi_{p}(x)\right| \leq \frac{1}{2}$, we may pass to the limit in (2.13) locally in space and conclude that $\tilde{\phi}_{\tilde{b}} \rightarrow \phi$ in $\mathcal{C}^{2, \alpha}$ and $\nu(\tilde{b}) \rightarrow \nu_{0}$ up to a subsequence as $\tilde{b} \rightarrow 0$. Moreover, (2.19) implies that $E_{\tilde{\phi}_{\tilde{b}}} \rightarrow E_{\phi}$ in $L^{2}\left(\mathbb{R}^{N}\right)$ and $\phi(r) \rightarrow 0$ as $r \rightarrow+\infty$ so that $\phi$ is a radial solution to

$$
\begin{aligned}
\Delta \phi & =G\left(-1-\nu_{0} \phi, 0\right)=\gamma_{N, p}\left(-1-\nu_{0} \phi\right)_{+}^{\frac{1}{p-1}+\frac{N}{N-2}}, \quad \phi(r) \rightarrow 0 \text { as } r \rightarrow+\infty, \\
\left|E_{\phi}\right|_{L^{2}} & =\left|E_{Q}\right|_{L^{2}}
\end{aligned}
$$

with $\gamma_{N, p}$ given by (1.17). From the scaling invariance of this equation, this implies

$$
\nu_{0}=1 \text { and } \phi=\phi_{Q}
$$

and thus from the uniqueness of the limit and (2.15)

$$
\left|\nabla \tilde{\phi}_{\tilde{b}}-\nabla \phi_{Q}\right|_{L^{2}}+\left|\tilde{\phi}_{\tilde{b}}-\phi_{Q}\right|_{\mathcal{e}^{2, \alpha}\left(\mathbb{R}^{N}\right)} \rightarrow 0 \text { and } \nu(\tilde{b}) \rightarrow 1 \text { as } \tilde{b} \rightarrow 0 .
$$

Note that this concludes the proof of (2.4). 
Step 4. Uniqueness.

Let

$$
\phi_{b}(x)=\nu(\tilde{b}) \tilde{\phi}_{\tilde{b}}\left(\frac{x}{\sqrt{\nu(\tilde{b})}}\right) \text { with } b=\frac{\tilde{b}}{\sqrt{\nu(\tilde{b})}} ;
$$

then from (2.20), $\phi_{b}$ is a smooth radially symmetric solution to

$$
\left\{\begin{array}{l}
\Delta \phi_{b}=G\left(-1-\phi_{b}, b\right)\left(x \chi_{p}(x)\right), \\
\left|\phi_{b}(0)-\phi_{Q}(0)\right|<\delta\left(b^{*}\right) \text { with } \delta\left(b^{*}\right) \rightarrow 0 \text { as } b^{*} \rightarrow 0, \\
\phi_{b}(r) \rightarrow 0 \text { as } r \rightarrow+\infty
\end{array}\right.
$$

Note that the cut-off does not see the rescaling from (2.20) and the definition (2.2) of $\chi_{p}$. Now let $\phi_{b_{1}}$ and $\phi_{b_{2}}$ be two smooth radial solutions of (2.22) with $\left|b_{1}\right|,\left|b_{2}\right|<b^{*}$ small enough; we claim

$$
\left|\nabla \phi_{b_{2}}-\nabla \phi_{b_{1}}\right|_{L^{2}} \leq C\left|b_{2}-b_{1}\right|
$$

Let us assume (2.23); then there is at most one smooth solution to (2.22). To prove the existence, it remains to prove from Steps 1, 2, 3 that any $b$ small enough may be written as $b=\frac{\tilde{b}}{\sqrt{\nu(\tilde{b})}}$. From (2.20), it suffices to prove that $\nu(\tilde{b})$ is a continuous function of $\tilde{b}$. Now from (2.21),

$$
\left|\nabla \phi_{b}\right|_{L^{2}}=(\nu(\tilde{b}))^{\frac{N+2}{4}}\left|\nabla \tilde{\phi}_{\tilde{b}}\right|_{L^{2}}=(\nu(\tilde{b}))^{\frac{N+2}{4}}\left|\nabla \phi_{Q}\right|_{L^{2}}
$$

and thus (2.23) implies

$$
\left|\left(\nu\left(\tilde{b}_{2}\right)\right)^{\frac{N+2}{4}}-\left(\nu\left(\tilde{b}_{1}\right)\right)^{\frac{N+2}{4}}\right| \leq C\left|\frac{\tilde{b}_{2}}{\sqrt{\nu\left(\tilde{b}_{2}\right)}}-\frac{\tilde{b}_{1}}{\sqrt{\nu\left(\tilde{b}_{1}\right)}}\right| .
$$

From (2.20), this implies

$$
\left|\nu\left(\tilde{b}_{2}\right)-\nu\left(\tilde{b}_{1}\right)\right| \leq C\left|\tilde{b}_{2}-\tilde{b}_{1}\right|
$$

for $\left|\tilde{b}_{1}, \tilde{b}_{2}\right|<b^{*}$ small enough, hence the continuity of the Lagrange multiplier.

Proof of (2.23). Let $b=b_{1}, b_{2}$. Observe from (2.20) that

$$
\left|\nabla \phi_{b}-\nabla \phi_{Q}\right|_{L^{2}}+\left|\phi_{b}-\phi_{Q}\right|_{e^{2, \alpha}\left(\mathbb{R}^{N}\right)} \leq \delta\left(b^{*}\right)
$$

with $\delta\left(b^{*}\right) \rightarrow 0$ as $b^{*} \rightarrow 0$.

Now (2.23) is a consequence of the spectral structure of the linearized operator of (2.22) close to $\phi_{Q}$. Indeed, let

$$
R=\phi_{b_{2}}-\phi_{b_{1}}
$$

then $R$ satisfies

$$
|\nabla R|_{L^{2}}+|R|_{\mathrm{e}^{2, \alpha}} \leq \delta\left(b^{*}\right)
$$

from (2.24) and the equation

$$
\Delta R+\frac{\partial G}{\partial a}\left(-1-\phi_{Q}, 0\right) R=H
$$


with

$$
\begin{aligned}
H= & G\left(-1-\phi_{b_{2}}, b_{2}\right)-G\left(-1-\phi_{b_{2}}, b_{1}\right)-\left(\frac{\partial G}{\partial a}\left(-1-\phi_{b_{1}}, b_{1}\right)-\frac{\partial G}{\partial a}\left(-1-\phi_{Q}, 0\right)\right) R \\
& +G\left(-1-\phi_{b_{1}}-R, b_{1}\right)-G\left(-1-\phi_{b_{1}}, b_{1}\right)+\frac{\partial G}{\partial a}\left(-1-\phi_{b_{1}}, b_{1}\right) R .
\end{aligned}
$$

We now recall the following spectral result, which has been proved in [27] as a consequence of the variational characterization of $Q$ that allows an explicit computation of the kernel of the Schrödinger operator $\Delta+\frac{\partial G}{\partial a}\left(-1-\phi_{Q}, 0\right)$.

Lemma 1 (Structure of the linear operator close to $\phi_{Q}$, 27]). There exists $c_{0}>0$ and a finite number of well localized eigenstates $\left(\psi_{j}\right)_{1 \leq j \leq J}$ corresponding to nonpositive eigenvalues $\left(\mu_{j}\right)_{1 \leq j \leq J}, \mu_{j}<0$, such that for all $\phi \in \dot{H}^{1}$ with radial symmetry,

$$
\int|\nabla \phi|^{2}-\frac{\partial G}{\partial a}\left(-1-\phi_{Q}, 0\right)|\phi|^{2} \geq c_{0} \int|\nabla \phi|^{2}-\frac{1}{c_{0}} \Sigma_{j=0}^{J}\left(\phi, \psi_{j}\right)^{2} .
$$

We now take the inner product of (2.26) with $\psi_{i}$ and $R$, integrate by parts and estimate the $H$ term. First observe from the uniform $L^{\infty}$ bounds on $\phi_{b}$ that the arguments of the nonlinearity $G$ lie in a uniform compact, so that we have from (2.24) and (2.25) a pointwise control:

$$
|H(x)| \leq C\left|b_{1}-b_{2}\right|+\delta\left(b^{*}\right)|R(x)| .
$$

Moreover, $\operatorname{Supp}(H) \subset\left\{r \leq 2 r_{Q}\right\}$ from (2.20) so that

$$
\begin{aligned}
|(H, R)|+\left|\left(H, \psi_{i}\right)\right|^{2} & \leq C\left|b_{2}-b_{1}\right||R|_{L^{2}\left(r \leq 2 r_{Q}\right)}+C\left(b_{1}-b_{2}\right)^{2}+\delta\left(b^{*}\right)|R|_{L^{2}\left(r \leq 2 r_{Q}\right)}^{2} \\
& \leq C\left|b_{2}-b_{1}\right||\nabla R|_{L^{2}}+C\left(b_{1}-b_{2}\right)^{2}+\delta\left(b^{*}\right)|\nabla R|_{L^{2}}^{2}
\end{aligned}
$$

where we used Poincaré's inequality. Inserting this into (2.28) yields:

$$
\begin{aligned}
c_{0} \int|\nabla R|^{2} & \leq\left(-\Delta R-\frac{\partial G}{\partial a}\left(-1-\phi_{Q}, 0\right) R, R\right)+C \Sigma_{j=1}^{J} \frac{\left(H, \psi_{j}\right)^{2}}{\mu_{j}^{2}} \\
& \leq C\left(b_{2}-b_{1}\right)^{2}+\frac{c_{0}}{2} \int|\nabla R|^{2}
\end{aligned}
$$

for $|b|<b^{*}$ small enough, and (2.23) is proved.

Step 5. Differentiability.

Observe that (2.23), (2.26) and the fact that $\operatorname{Supp}(H) \subset\left\{r \leq 2 r_{Q}\right\}$ ensure from standard elliptic regularity a uniform control

$$
|R|_{\mathcal{C}^{2, \alpha}\left(\mathbb{R}^{N}\right)} \leq C\left|b_{2}-b_{1}\right|
$$

Then let

Then $J$ satisfies

$$
J=\frac{R}{b_{2}-b_{1}}
$$

$$
\begin{gathered}
\Delta J+\frac{\partial G}{\partial a}\left(-1-\phi_{b_{1}}, b_{1}\right) J=\frac{G\left(-1-\phi_{b_{2}}, b_{2}\right)-G\left(-1-\phi_{b_{2}}, b_{1}\right)}{b_{2}-b_{1}} \\
+\frac{1}{b_{2}-b_{1}}\left[G\left(-1-\phi_{b_{1}}-R, b_{1}\right)-G\left(-1-\phi_{b_{1}}, b_{1}\right)+\frac{\partial G}{\partial \phi}\left(-1-\phi_{b_{1}}, b_{1}\right) R\right] .
\end{gathered}
$$


We now pass to the limit as $b_{2} \rightarrow b_{1}$. First observe from the regularity of $G$ and $\phi_{b}$ that

$$
\frac{G\left(-1-\phi_{b_{2}}, b_{2}\right)-G\left(-1-\phi_{b_{2}}, b_{1}\right)}{b_{2}-b_{1}} \rightarrow \frac{\partial G}{\partial b}\left(-1-\phi_{b_{1}}, b_{1}\right) \text { in } \quad L^{\infty} \quad \text { as } \quad b_{2} \rightarrow b_{1} .
$$

For the nonlinear term, we use the $\mathcal{C}^{2, \alpha}$ regularity of $G$ in $-1-\phi_{b}$ which ensures the pointwise control

$$
\begin{aligned}
& \frac{1}{\left|b_{2}-b_{1}\right|}\left|G\left(-1-\phi_{b_{1}}-R, b_{1}\right)-G\left(-1-\phi_{b_{1}}, b_{1}\right)+\frac{\partial G}{\partial a}\left(-1-\phi_{b_{1}}, b_{1}\right) R\right| \\
\leq & \frac{C}{b_{2}-b_{1}}|R(x)|^{2} \leq C\left|b_{2}-b_{1}\right|
\end{aligned}
$$

from (2.29). We thus conclude from (2.29) that, up to a subsequence, we have $J \rightarrow K$ as $b_{2} \rightarrow b_{1}$ in $\mathcal{C}_{l o c}^{2, \alpha}$ where $|\nabla K|_{L^{2}} \leq C$ from (2.23) and $K$ satisfies

$$
\Delta K+\frac{\partial G}{\partial a}\left(-1-\phi_{b_{1}}, b_{1}\right) K=\frac{\partial G}{\partial b}\left(-1-\phi_{b_{1}}, b_{1}\right) \text {. }
$$

The uniqueness of $K$ follows from the invertibility of $\Delta+\frac{\partial G}{\partial a}\left(-1-\phi_{b_{1}}, b_{1}\right)$ on the subset of $\dot{H}^{1}$ restricted to radially symmetric distributions. This invertibility property indeed follows from the Lax Milgram theorem and (2.28) for $\Delta+$ $\frac{\partial G}{\partial \phi}\left(-1-\phi_{Q}, 0\right)$ and then holds again for $\Delta+\frac{\partial G}{\partial a}\left(-1-\phi_{b_{1}}, b_{1}\right)$ from standard perturbation theory using (2.20). Eventually, the $K$ equation whose right-hand side is compactly supported, the regularity of $G$ and standard elliptic regularity estimates ensure (2.5), while (2.6) follows from the explicit computation $\frac{\partial G}{\partial b}\left(-1-\phi_{Q}, 0\right)=0$.

This concludes the proof of Proposition 2 .

We now exhibit the self-similar profile $Q_{b}$ solution to the self-similar equation (1.21) as an explicit function of $\phi_{b}$ :

Proposition 3 (Derivation and properties of the exact self-similar profiles). Under the hypothesis of Proposition 2, let

$$
Q_{b}(x, v)=\left(-\frac{|v|^{N-2}}{N-2}-1-b \chi_{p}(x) x \cdot v-\phi_{b}\right)_{+}^{\frac{1}{p-1}}
$$

then $Q_{b} \in \mathcal{E}_{r}$ and is uniformly compactly supported in $(x, v)$ with $\operatorname{Supp}\left(Q_{b}\right) \rightarrow$ $\operatorname{Supp}(Q)$ as $b \rightarrow 0$, and

$Q_{b}$ is an exact solution to the self-similar equation (1.21).

Moreover, the flow map

$$
b \rightarrow Q_{b} \quad \text { is } \mathcal{C}^{0}\left(\left(-b^{*}, b^{*}\right), W^{1,1} \cap \mathcal{C}^{0}\right) \cap \mathcal{C}^{1}\left(\left(-b^{*}, b^{*}\right), L^{1}\right),
$$

and the following properties hold:

(i) $Q_{b}$ is a deformation of $Q$ :

$$
Q_{b}(b=0)=Q \text { and } \frac{\partial Q_{b}}{\partial b}(b=0)=|v|^{4-N} x \cdot \nabla_{v} Q .
$$

(ii) $Q_{b}$ has zero energy: let $\mathcal{H}_{N}$ be the homogeneous Hamiltonian (1.15); then

$$
\mathcal{H}_{N}\left(Q_{b}\right)=0 \text {. }
$$

(iii) $Q_{b}$ has super critical mass:

$$
\int_{\mathbb{R}^{2 N}} Q_{b}+\frac{1}{p} \int_{\mathbb{R}^{2 N}} Q_{b}^{p}=\int_{\mathbb{R}^{2 N}} Q+\frac{1}{p} \int_{\mathbb{R}^{2 N}} Q^{p}+\frac{b^{2}}{2}\left(m^{*}+o(1)\right) \text { as } b \rightarrow 0
$$


with

$$
m^{*}=-\int|v|^{4-N}(x \cdot v) x \cdot \nabla_{v} Q=\left\{\begin{array}{l}
\int|x|^{2} Q \text { for } N=4 \\
\int Q\left(|x|^{2}|v|+\frac{(x \cdot v)^{2}}{|v|}\right) \text { for } N=3
\end{array}\right.
$$

Proof of Proposition 3 and Proposition 1 . Let $Q_{b}$ given by (2.30); then $Q_{b}$ is radially symmetric and uniformly compactly supported from (2.4). Moreover, from the definition (2.2) and (2.4), we have the fundamental property

$$
\chi_{p}=1 \text { on } \operatorname{Supp}\left(Q_{b}\right),
$$

and (2.31) follows. The regularity (2.32) of the flow map $b \rightarrow Q_{b}$ is a direct consequence of the definition (2.30) and the regularity (2.5). It remains to prove (2.33). We rewrite (2.30) as

$$
\frac{|v|^{N-2}}{N-2}+1+b x \cdot v+\phi_{b}+Q_{b}^{p-1}=0 \text { on } \operatorname{Supp}\left(Q_{b}\right),
$$

differentiate this expression with respect to $b$ and estimate the result at $b=0$. We get using (2.6):

$$
\frac{\partial Q_{b}}{\partial b}(b=0)=-\frac{x \cdot v}{(p-1) Q^{p-2}} .
$$

We now rewrite the $Q$ equation $\frac{|v|^{N-2}}{N-2}+1+\phi_{Q}+Q^{p-1}=0$, differentiate it with respect to $v$ and take the inner product of the result with $x$ to get

$$
-\frac{x \cdot v}{(p-1) Q^{p-2}}=|v|^{4-N} x \cdot \nabla_{v} Q
$$

and (2.33) is proved.

Now (2.34) follows from Pohozaev identity that is obtained by multiplying (1.21) by $x \cdot v$ and integrating by parts. To prove (2.35), we compute from (2.30) and (2.32):

$$
\begin{aligned}
\frac{d}{d b}\left\{\int_{\mathbb{R}^{2 N}} Q_{b}+\frac{1}{p} \int_{\mathbb{R}^{2 N}} Q_{b}^{p}\right\} & =\int_{\mathbb{R}^{2 N}} \frac{\partial Q_{b}}{\partial b}\left(1+Q_{b}^{p-1}\right) \\
& =-\int_{\mathbb{R}^{2 N}} \frac{\partial Q_{b}}{\partial b}\left(b x \cdot v+\frac{|v|^{N-2}}{N-2}+\phi_{b}\right) .
\end{aligned}
$$

Let us now differentiate (2.34) with respect to $b$ to get

$$
\int_{\mathbb{R}^{2 N}} \frac{\partial Q_{b}}{\partial b}\left(\frac{|v|^{N-2}}{N-2}+\phi_{b}\right)=0
$$

and thus from (2.33)

$$
\begin{aligned}
\frac{d}{d b}\left\{\int_{\mathbb{R}^{2 N}} Q_{b}+\frac{1}{p} \int_{\mathbb{R}^{2 N}} Q_{b}^{p}\right\} & =-b \int_{\mathbb{R}^{2 N}} \frac{\partial Q_{b}}{\partial b} x \cdot v \\
& =-b\left(\int_{\mathbb{R}^{2 N}}(x \cdot v)|v|^{4-N} x \cdot \nabla_{v} Q+o(1)\right)
\end{aligned}
$$

and (2.35) now follows by integrating in $b$ and the definition (2.36).

This concludes the proof of Proposition 3 which implies Proposition 1. 
2.2. Nonlinear decomposition close to $Q$. In this section we introduce, using modulation theory, a geometrical decomposition of the functions $f \in \mathcal{E}$ with spherical symmetry close in some sense to the two dimensional manifold

$$
\mathcal{M}=\left\{Q_{b}\left(\frac{x}{\lambda}, \lambda v\right), \quad b \in\left(-b^{*}, b^{*}\right), \quad \lambda>0\right\} .
$$

Before we detail the decomposition, let us recall the following elementary result of decay for the gravitational field in the radial case:

Lemma 2 ( $L^{\infty}$ estimate on the gravitational field in the radial case). Let $N=3,4$, $p \in(2,+\infty)$ and $f \in \mathcal{E}$ with spherical symmetry; then

$$
\forall r>0, \quad 0 \leq \sigma_{N} r^{N-1} E_{f}(r) \leq|f|_{L^{1}}, \quad \sigma_{N}(N-2) r^{N-2} \phi_{f}(r) \geq-|f|_{L^{1}} .
$$

Proof of Lemma 2. We may assume $f$ smooth and then conclude by density. The assumptions on $f$ imply that $\rho_{f} \geq 0$ and has radial symmetry. We then integrate the Poisson kernel in radial coordinates and get for $r>0$ :

$$
\begin{gathered}
E_{f}(r)=\frac{1}{r^{N-1}} \int_{0}^{r} u^{N-1} \rho_{f}(u) d u \leq \frac{|f|_{L^{1}}}{\sigma_{N} r^{N-1}} \\
-\phi_{f}(r)=\int_{r}^{+\infty} E_{f}(\tau) d \tau \leq \frac{|f|_{L^{1}}}{\sigma_{N}} \int_{r}^{+\infty} \frac{d \tau}{\tau^{N-1}}=\frac{|f|_{L^{1}}}{\sigma_{N}(N-2) r^{N-2}}
\end{gathered}
$$

and (2.38) follows. This concludes the proof of Lemma 2 .

Let us introduce the following smooth regularization of the characteristic function of respectively the regions $|x||v| \leq\left(4|Q|_{L^{1}} / \sigma_{N}\right)^{\frac{1}{N-2}}$ and $\operatorname{Supp}(Q)$ :

$$
\psi(x, v)=\psi(|x||v|) \text { where } \psi(\tau)=\left\{\begin{array}{l}
1 \text { for } 0 \leq \tau \leq\left[\frac{4|Q|_{L^{1}}}{\sigma_{N}}\right]^{\frac{1}{N-2}}, \\
0 \text { for } \tau \geq\left[\frac{8|Q|_{L^{1}}}{\sigma_{N}}\right]^{\frac{1}{N-2}}
\end{array}\right.
$$

with

$$
\psi^{\prime} \leq 0
$$

and

$$
\zeta(x, v)=\left\{\begin{array}{l}
1 \text { for } \frac{|v|^{N-2}}{N-2}+1+\phi_{Q} \leq \frac{1}{4}, \\
0 \text { for } \frac{|v|^{N-2}}{N-2}+1+\phi_{Q} \geq \frac{1}{2} .
\end{array}\right.
$$

Observe from Lemma 2 that

$$
\frac{|v|^{N-2}}{N-2}+\phi_{Q}(r) \geq \frac{1}{(N-2) r^{N-2}}\left((r|v|)^{N-2}-\frac{|Q|_{L^{1}}}{\sigma_{N}}\right)
$$

and thus (1.18) implies

$$
\psi(x, v)=\zeta(x, v)=1 \text { on } \operatorname{Supp}(Q) .
$$

We now claim the following nonlinear decomposition property:

Proposition 4 (Nonlinear decomposition of the solution). Let $N=3,4$ and $p \in(2,+\infty)$. There exists a universal constant $\alpha^{*}=\alpha^{*}(N, p)>0$ such that the following holds true. Let $f \in \mathcal{E}$ with radial symmetry and assume the following:

(i) Proximity to $Q$ in $L^{1}, L^{p}$ size:

$$
\left.|| f\right|_{L^{1}}-|Q|_{L^{1}}|+||f|_{L^{p}}-|Q|_{L^{p}} \mid<\alpha^{*} .
$$


(ii) $f$ has a small renormalized Hamiltonian: it holds that

$$
\lambda_{f}^{N-2} \mathcal{H}(f)<\alpha^{*}
$$

with $\mathcal{H}(f)$ given by (1.6) and

$$
\lambda_{f}=\left(\frac{\left.\left.|| v\right|^{N-2} Q\right|_{L^{1}}}{\|\left.\left. v\right|^{N-2} f\right|_{L^{1}}}\right)^{\frac{1}{N-2}} .
$$

Then there exists $\lambda>0, b \in \mathbb{R}$, such that $f$ admits a nonlinear geometrical decomposition

$$
f(x, v)=\left(Q_{b}+\varepsilon\right)\left(\frac{x}{\lambda}, \lambda v\right)
$$

where $\varepsilon$ satisfies the orthogonality conditions

$$
\int_{\mathbb{R}^{2 N}}(x \cdot v) \psi \varepsilon=0, \quad \int_{\mathbb{R}^{2 N}}|v|^{4-N}|x|^{2} \zeta \varepsilon=0,
$$

and the following uniform control on the geometrical decomposition holds:

$$
\left|\frac{\lambda}{\lambda_{f}}-1\right|+|b|+|\varepsilon|_{\varepsilon}<\delta\left(\alpha^{*}\right)
$$

where $\delta\left(\alpha^{*}\right) \rightarrow 0$ as $\alpha^{*} \rightarrow 0$.

Proof of Proposition 4. Proposition 4 is a standard consequence of the variational characterization of $Q$ and the implicit function theorem. Let $\mathcal{H}_{N}(f)$ be the homogeneous Hamiltonian of (1.14) given by (1.15) and let

$$
g(x, v)=f\left(\lambda_{f} x, \frac{v}{\lambda_{f}}\right) .
$$

Then from (2.43), (2.45) and (2.44), we have

$$
\left.|| g\right|_{L^{1}}-|Q|_{L^{1}}|+||g|_{L^{p}}-\left.|Q|_{L^{p}}\left|+\mathcal{H}_{N}(g)<2 \alpha^{*}, \quad\right||v|^{2} g\right|_{L^{1}}=\left.\left.|| v\right|^{2} Q\right|_{L^{1}} .
$$

From the variational characterization of $Q$ (see [26, [27]) and $g \in \mathcal{E}$ with radial symmetry, this implies from standard argument (see for example Proposition 5.1 in [26]) that

$$
|g-Q|_{\varepsilon}<\delta\left(\alpha^{*}\right) \text { with } \delta\left(\alpha^{*}\right) \rightarrow 0 \text { as } \alpha^{*} \rightarrow 0 .
$$

For $\alpha>0$, let $U_{\alpha}=\left\{g \in \mathcal{E}\right.$ with $\left.|g-Q|_{\mathcal{\varepsilon}}<\alpha\right\}$. We claim that there exists $\alpha^{*}>0$ and a unique $\mathcal{C}^{1}$ map $U_{\alpha^{*}} \rightarrow\left(1-\lambda^{*}, 1+\lambda^{*}\right) \times\left(-b^{*}, b^{*}\right)$ such that for all $g \in U_{\alpha^{*}}$, there is a unique $\left(\lambda_{1}, b_{1}\right)$ such that

$$
\varepsilon_{\lambda_{1}, b_{1}, g}=g\left(\frac{x}{\lambda_{1}}, \lambda_{1} v\right)-Q_{b_{1}}
$$

satisfies the orthogonality conditions (2.47); moreover,

$$
\left|\varepsilon_{\lambda_{1}, b_{1}, g}\right|_{\mathcal{E}}+\left|\lambda_{1}-1\right|+\left|b_{1}\right| \leq C \alpha^{*} .
$$

Indeed, we define the functionals

$$
F_{1}\left(\lambda_{1}, b_{1}, g\right)=\int_{\mathbb{R}^{2 N}}(x \cdot v) \psi \varepsilon_{\lambda_{1}, b_{1}, g}=0, \quad F_{2}\left(\lambda_{1}, b_{1}, g\right)=\int_{\mathbb{R}^{2 N}}|v|^{4-N}|x|^{2} \zeta \varepsilon_{\lambda_{1}, b_{1}, g}=0
$$


and compute from (2.32), (2.33), (2.42) and (2.36) at $\left(\lambda_{1}=1, b_{1}=0, g=Q\right)$ :

$$
\begin{gathered}
\frac{\partial F_{1}}{\partial \lambda_{1}}=-\int_{\mathbb{R}^{2 N}}\left(x \cdot \nabla_{x} Q-v \cdot \nabla_{v} Q\right)(x \cdot v) \psi=-\int_{\mathbb{R}^{2 N}}\left(x \cdot \nabla_{x} Q-v \cdot \nabla_{v} Q\right)(x \cdot v)=0, \\
\frac{\partial F_{1}}{\partial b_{1}}=-\int_{\mathbb{R}^{2 N}}|v|^{4-N} x \cdot \nabla_{v} Q(x \cdot v) \psi=m^{*}>0 \\
\frac{\partial F_{2}}{\partial \lambda_{1}}=-\int_{\mathbb{R}^{2 N}}\left(x \cdot \nabla_{x} Q-v \cdot \nabla_{v} Q\right)|x|^{2}|v|^{4-N} \zeta=(N-2) \int_{\mathbb{R}^{2 N}}|x|^{2}|v|^{4-N} Q \\
\frac{\partial F_{2}}{\partial b_{1}}=-\int_{\mathbb{R}^{2 N}}|v|^{2(4-N)} x \cdot \nabla_{v} Q|x|^{2} \zeta=0
\end{gathered}
$$

where the last computation follows from the symmetry properties of $Q$. The Jacobian of the functional $\left(F_{1}, F_{2}\right)$ is nonzero and thus the implicit function theorem applies and yields the claim. This concludes the proof of Proposition 4 .

\section{Stability OF THE SELF-Similar BLOW UP DYNAMiCS}

This section is devoted to the proof of the main theorem, Theorem 3 The global argument is a bootstrap argument. In section 3.1, we give an explicit geometrical description of the set of initial data which led to the self-similar blow up solutions described by Theorem 3. This decomposition is based on Proposition 4, In order to control the geometrical parameters associated to this decomposition, we use two major informations: a virial dispersive estimate which is the heart of our analysis and which implies both the existence of a Lyapounov function in time and a dispersive control of the solution outside the origin (see Lemma 4 of section 3.2); the coercivity of the linearized energy which implies uniform controls on the solution in the vicinity of the ground state as proved in section 3.3. This allows us to close the bootstrap argument (see section 3.4), and the obtained estimates then imply Theorem 33 see section 3.5.

3.1. Setting of the bootstrap. Let an initial $f_{0} \in \mathcal{C}_{0, \text { rad }}^{1}$ satisfying the assumptions of Theorem 3 . Let $f(t)$ be the corresponding classical solution to (1.3), (1.14) with life time $0<T \leq+\infty$. First observe from (1.25), Proposition 3 and the regularity of the flow $f \in \mathrm{e}^{0}([0, T), \mathcal{E})$ that there exists $t_{0} \in[0, T]$ such that for all $t \in\left[0, t_{0}\right), f(t)$ satisfies (2.43) and (2.44) provided $b^{*}>0$ in Theorem 3 has been chosen small enough. Thus $f(t)$ admits from Proposition 4 a nonlinear decomposition

$$
f(t, x, v)=\left(Q_{b(t)}(x, v)+\varepsilon\right)\left(t, \frac{x}{\lambda(t)}, \lambda(t) v\right)
$$

where $\varepsilon(t)$ satisfies the orthogonality conditions (2.47). Note also that $b(t), \lambda(t)$ are for each given $t$ given by the implicit function theorem and thus smooth functions of $f(t)$ for the $\mathcal{E}$ norm. From the regularity $f(t) \in \mathcal{C}^{1}([0, T), \mathcal{E})$, we conclude that $b(t), \lambda(t)$ are $\mathcal{C}^{1}$ functions of time. Let us define the rescaled time

$$
s(t)=s_{0}+\int_{0}^{t} \frac{d \tau}{\lambda^{N-2}(\tau)}
$$

with

$$
s_{0}=-\frac{\log (\lambda(0))}{b(0)} \geq 1
$$


provided $\lambda\left(b_{0}\right)>0$ has been chosen small enough in Theorem 3. Let

$$
\begin{gathered}
K_{b}=\operatorname{Supp}\left(Q_{b}\right)=\left\{(x, v) \text { with } \frac{|v|^{N-2}}{N-2}+b x \cdot v+1+\phi_{b}(x) \leq 0\right\}, \\
\kappa(t, x, v)=\left(\frac{|v|^{N-2}}{N-2}+b \chi(x) x \cdot v+1+\phi_{b}\right) \mathbf{1}_{x \notin K_{b}} \geq 0 .
\end{gathered}
$$

Let $\delta_{0}>0$ be the universal constant in the coercivity estimate (3.53); then there exists $0<\eta, c \ll 1$ such that

$$
\forall|x| \leq \eta, \quad\left|(1+x)^{p}-1-p x-\frac{p(p-1)}{2} x^{2}\right| \leq \frac{\delta_{0}}{100}|x|^{2},
$$

and

$$
\forall|x| \geq \eta \quad \text { with } \quad x \geq-1, \quad(1+x)^{p}-1-p x \geq c|x|^{p} .
$$

We then split $\varepsilon(t)$ into three terms:

$$
\varepsilon=\varepsilon \mathbf{1}_{(x, v) \notin K_{b}}+\varepsilon \mathbf{1}_{(x, v) \in K_{b}} \mathbf{1}_{|\varepsilon(x)| \leq \eta Q_{b}(x)}+\varepsilon \mathbf{1}_{(x, v) \in K_{b}} \mathbf{1}_{|\varepsilon(x)| \geq \eta Q_{b}(x)}=\varepsilon_{1}+\varepsilon_{2}+\varepsilon_{3} .
$$

Observe now that we may have chosen $\lambda\left(b_{0}\right), \alpha\left(b_{0}\right)$ in Theorem 3 small enough so that the following controls hold at $t=0$ :

$$
\begin{gathered}
\int_{\mathbb{R}^{2 N}} \kappa \varepsilon_{1}(0)+\int_{\mathbb{R}^{2 N}} \varepsilon_{1}^{p}(0)+\int_{\mathbb{R}^{2 N}} \varepsilon_{2}^{2}(0) Q_{b(0)}^{p-2}+\int_{\mathbb{R}^{2 N}}\left|\varepsilon_{3}(0)\right|^{p}<b^{4}(0), \\
\lambda_{0}\left(1+\left|\mathcal{H}\left(f_{0}\right)\right|\right)^{10}<b^{30}(0) .
\end{gathered}
$$

Our main claim is that the two estimates (3.9) and (3.10) which precise the proximity of the initial data to the specific self-similar solution $Q_{b_{0}}$-and not only $Q_{-}$ imply a trapping of the flow meaning that the solution $f(t)$ must remain close to the manifold $\mathcal{M}$ given by (2.37). The heart of our analysis is the following dynamical -and no longer variational- bootstrap argument:

Lemma 3 (Bootstrap). Assume (3.3), (3.9) and (3.10) at time $t=0$. Let $0<$ $t_{1} \leq T$ such that: $\forall t \in\left[0, t_{1}\right)$, it holds that

$$
\begin{gathered}
\int_{\mathbb{R}^{2 N}} \kappa \varepsilon_{1}(t)+\int_{\mathbb{R}^{2 N}} \varepsilon_{1}^{p}(t)+\int_{\mathbb{R}^{2 N}} \varepsilon_{2}^{2}(t) Q_{b}^{p-2}+\int_{\mathbb{R}^{2 N}}\left|\varepsilon_{3}(t)\right|^{p}<b(t), \\
\lambda(t) \leq 10 \lambda(0), \\
\forall s \in\left[s_{0}, s_{1}=s\left(t_{1}\right)\right), \quad \lambda(s) \leq e^{-\frac{b(0)}{5} s}, \\
\frac{b(0)}{2} \leq b(t) \leq 2 b(0) .
\end{gathered}
$$

Then it holds that $\forall t \in\left[0, t_{1}\right)$,

$$
\begin{gathered}
\int_{\mathbb{R}^{2 N}} \kappa \varepsilon_{1}(t)+\int_{\mathbb{R}^{2 N}} \varepsilon_{1}^{p}(t)+\int_{\mathbb{R}^{2 N}} \varepsilon_{2}^{2}(t) Q_{b}^{p-2}+\int_{\mathbb{R}^{2 N}}\left|\varepsilon_{3}(t)\right|^{p}<\delta(b(0)) b^{2}(t), \\
\lambda(t) \leq \lambda(0), \\
b(0)(1-\delta(b(0))) \leq b(t) \leq b(0)(1+\delta(b(0))) \\
\lambda(s) \leq e^{-\frac{b(0)}{2} s}
\end{gathered}
$$

where $\delta(b(0)) \rightarrow 0$ as $b(0) \rightarrow 0$. 
The rest of the paper in organized as follows. In section 3.2, we prove the virial estimate (3.21) of Lemma 4 which implies the monotonicity (3.22) and hence the nondegeneracy of the self-similar parameter $b$. We then inject this monotonicity into the conservation of the Hamiltonian and derive from the coercivity properties of the linearized Casimir functional uniform bounds on the excess of mass, Lemma 5 . In section 3.3, we inject these estimates into the modulation equation governing the geometrical parameters to finish the proof of Lemma 3 Eventually, we conclude in section 3.5 the proof of Theorem 3 mainly as a consequence of the bootstrap estimates of Lemma 3 .

3.2. The virial dispersive estimate and monotonicity of $b$. We start with the fundamental tool of our analysis which is a local dispersive structure inherited from the virial law (1.11). Moreover, the corresponding estimate directly implies the existence of a Lyapounov function which is essentially the finite dimensional projection $b$.

Before stating the result, let us make a few heuristic comments. Let $\varepsilon$ be given by the geometrical decomposition (3.1); then $\varepsilon$ is governed to leading order by the linearized flow close to $Q$ :

$$
\partial_{s} \varepsilon+\mathcal{L} \varepsilon \sim 0 \text { with } \mathcal{L} \varepsilon=v \cdot \nabla_{x} \varepsilon-E_{Q} \cdot \nabla_{v} \varepsilon-E_{\varepsilon} \cdot \nabla_{v} Q .
$$

The dispersive structure of the linearized operator $\mathcal{L}$ has been completely understood in [27] using in particular the strategy developed by Weinstein [45]. Roughly speaking, this operator displays algebraic instabilities on the support of $Q$ generated by the large group of symmetries and which are controlled in our setting thanks to the choice of orthogonality conditions (2.47). Outside the support of $Q$, the linear flow reduces to the transport by the field $E_{Q}$ whose characteristics are explicit and coincide with the level set of the microscopic energy

$$
e_{Q}(x, v)=\frac{|v|^{N-2}}{N-2}+\phi_{Q}(|x|) .
$$

Note that these characteristics are trapped, i.e. compact in $(x, v)$ for $e_{Q}<0$, hence no dispersion occurs, and nontrapped for $e_{Q}>0$, hence the flow enjoys standard linear dispersive estimates; see 27.

Let the cut-off $\psi$ be given by (2.39); then we may hope from (3.19) a dispersive estimate for $\varepsilon$ in the region $e_{Q}>0$ which contains $\operatorname{Supp}(1-\psi)$. Figure 1 illustrates the nondispersive and dispersive regions in the phase space. Nevertheless, the typical space time smoothing effect for the linear flow (3.19) yields a local in space only dispersive estimate of the form

$$
\int_{0}^{+\infty} \int_{|x| \leq C}(1-\psi)|v|^{N-2} \varepsilon(s) d s<C \sup _{s \geq 0}|\varepsilon(s)|_{\varepsilon} .
$$

Our fundamental observation is that for the full nonlinear equation, the global virial law (1.11) together with our specific choice of orthogonality condition (2.47) implies a global in space dispersive estimate for $\varepsilon$ in the dispersive zone Supp $(1-\psi)$. Indeed, we claim:

Lemma 4 (Virial dispersion and monotonicity of $b$ ). We have the following dispersive estimate: $\forall s \in\left[s_{0}, s_{1}\right)$,

$$
b_{s} \geq C\left[\int(1-\psi)|v|^{N-2} \varepsilon-\sqrt{\lambda}\left(1+\left|\mathcal{H}\left(f_{0}\right)\right|\right)\right]
$$




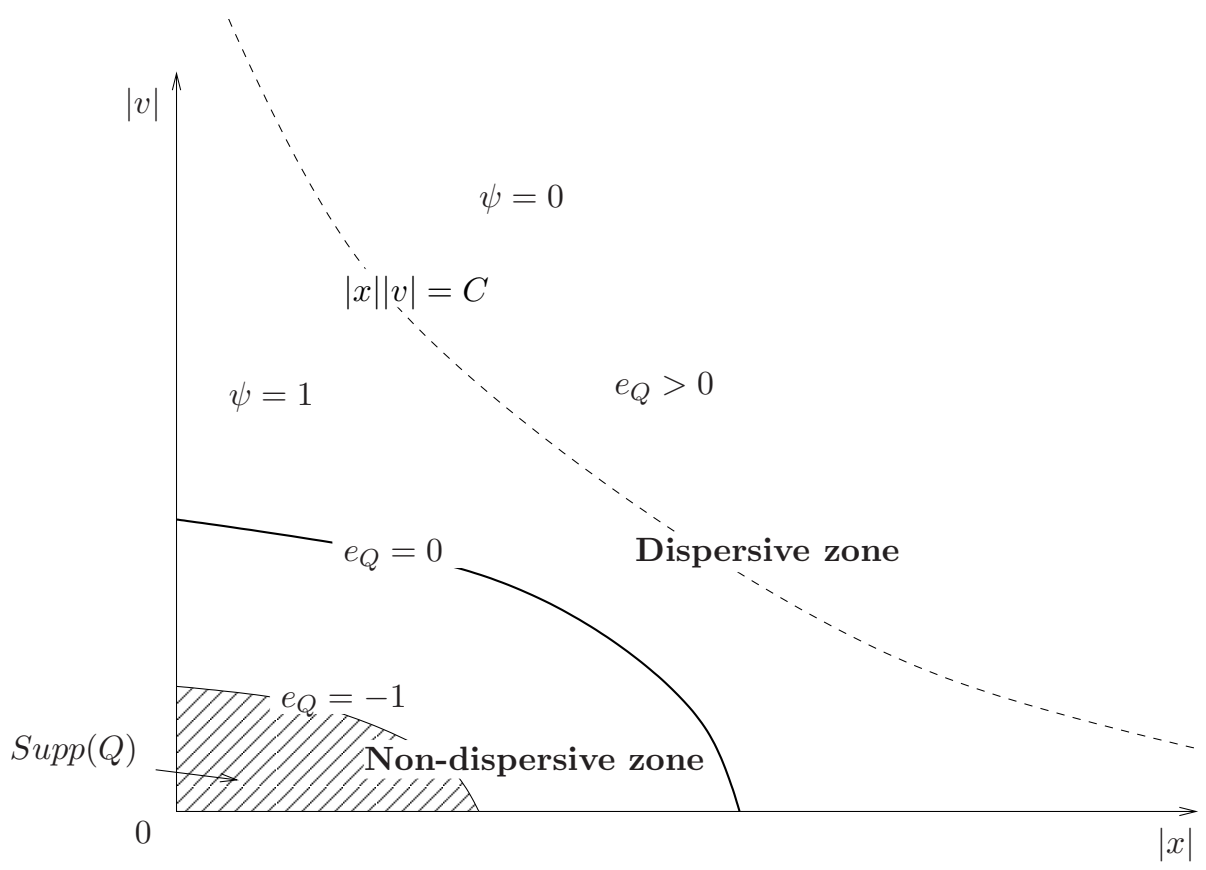

FiguRE 1. Dispersive and nondispersive regions

for some universal constant $C=C(N, p)>0$. Moreover, $b$ is almost nondecreasing and it holds that

$$
b(s) \geq b(0)(1-\delta(b(0)))
$$

where $\delta(b(0)) \rightarrow 0$ as $b(0) \rightarrow 0$.

Remark 2. Assume that (3.21) holds for all time $s \geq s_{0}$; then the boundedness of $b$ and the control of the scaling parameter (3.13) imply the global dispersive estimate

$$
\int_{s_{0}}^{+\infty} \int_{\mathbb{R}^{2 N}}(1-\psi)|v|^{N-2} \varepsilon(s) d s<+\infty
$$

for the nonlinear problem which is a nonlinear global in space improvement of the linear estimate (3.20).

Proof of Lemma 4. Step 1. The virial dispersive estimate.

For the sake of clarity, we distinguish the cases $N=3$ and $N=4$.

Case $N=4$.

Let $\psi$ be the cut-off function (2.39); we then compute from (1.14)

$$
\begin{aligned}
-\frac{d}{d t} & \left(\int_{\mathbb{R}^{2 N}}(x \cdot v) \psi(x, v) f(t, x, v) d x d v\right) \\
& =-\int_{\mathbb{R}^{2 N}} f \psi\left(|v|^{2}-x \cdot E_{f}\right)-\int_{\mathbb{R}^{2 N}}(x \cdot v) f\left(v \cdot \nabla_{x} \psi-E_{f} \cdot \nabla_{v} \psi\right) .
\end{aligned}
$$


We now use the conservation of the Hamiltonian

$$
2 \mathcal{H}\left(f_{0}\right)=\int_{\mathbb{R}^{2 N}}|v|^{2} f-\int\left|E_{f}\right|^{2}=\int_{\mathbb{R}^{2 N}}|v|^{2} f-\int_{\mathbb{R}^{2 N}} f x \cdot E_{f}
$$

and Pohozaev identity to derive

$$
\begin{aligned}
-\frac{d}{d t}\left(\int_{\mathbb{R}^{2 N}}(x \cdot v) \psi f\right)= & \int_{\mathbb{R}^{2 N}} f(1-\psi)\left(|v|^{2}-x \cdot E_{f}\right)-2 \mathcal{H}\left(f_{0}\right) \\
& -\int_{\mathbb{R}^{2 N}}(x \cdot v) f\left(v \nabla_{x} \psi-E_{f} \cdot \nabla_{v} \psi\right) .
\end{aligned}
$$

We now rescale this relation according to

$$
f(t, x, v)=g\left(s, \frac{x}{\lambda(t)}, \lambda(t) v\right)=\left(Q_{b(s)}+\varepsilon\right)\left(s, \frac{x}{\lambda(s)}, \lambda(s) v\right)
$$

where the rescaled time is given by (3.2). Remark that $\psi$ is invariant through this transformation from its definition (2.39). We get

$$
\begin{aligned}
-\frac{d}{d s}\left(\int_{\mathbb{R}^{2 N}}(x \cdot v) \psi g\right) & =\int(1-\psi) g\left(|v|^{2}-x \cdot E_{g}\right)-2 \lambda^{2} \mathcal{H}\left(f_{0}\right) \\
& -\int_{\mathbb{R}^{2 N}}(x \cdot v) g\left(v \cdot \nabla_{x} \psi-E_{g} \cdot \nabla_{v} \psi\right) .
\end{aligned}
$$

Observe from (3.9) that $|g|_{L^{1}}=|f|_{L^{1}}=\left|f_{0}\right|_{L^{1}} \leq 2|Q|_{L^{1}}$ and thus (2.38) and (2.39) imply for $(x, v) \in \operatorname{Supp}(1-\psi)$ :

$$
\begin{aligned}
|v|^{2}-x \cdot E_{g} & =\frac{|v|^{2}}{2}+\frac{1}{|x|^{2}}\left(\frac{|x|^{2}|v|^{2}}{2}-|x|^{3} E_{g}(|x|)\right) \\
& \geq \frac{|v|^{2}}{2}+\frac{1}{|x|^{2}}\left(\frac{|x|^{2}|v|^{2}}{2}-\frac{|f|_{L^{1}}}{\sigma_{4}}\right) \\
& \geq \frac{|v|^{2}}{2}+\frac{1}{\sigma_{4}|x|^{2}}\left(2|Q|_{L^{1}}-|f|_{L^{1}}\right) \geq \frac{|v|^{2}}{2} .
\end{aligned}
$$

Moreover, we have from the very specific choice of $\psi(2.39)$ and (2.40):

$-\int_{\mathbb{R}^{2 N}}(x \cdot v) g\left(v \cdot \nabla_{x} \psi-E_{g} \cdot \nabla_{v} \psi\right)=-2 \int_{\mathbb{R}^{2 N}} g \frac{(x \cdot v)^{2}}{|x||v|}\left(|v|^{2}-x \cdot E_{g}\right) \psi^{\prime}(|x||v|) \geq 0$

from (3.26) and $\operatorname{Supp}\left(\psi^{\prime}\right) \subset \operatorname{Supp}(1-\psi)$. Inserting (3.26) and (3.27) into (3.25) and using (2.42) yields:

$$
-\frac{d}{d s}\left(\int_{\mathbb{R}^{2 N}}(x \cdot v) \psi g\right) \geq \frac{1}{2} \int_{\mathbb{R}^{2 N}}(1-\psi)|v|^{2} \varepsilon-\lambda^{2} \mathcal{H}\left(f_{0}\right) .
$$

We now have from our choice of orthogonality condition (2.47):

$$
\int_{\mathbb{R}^{2 N}}(x \cdot v) \psi g=\int_{\mathbb{R}^{2 N}}(x \cdot v) Q_{b}
$$

and thus from (2.33) and (2.36):

$$
\begin{gathered}
-\frac{d}{d s}\left(\int_{\mathbb{R}^{2 N}}(x \cdot v) \psi g\right)=-\frac{d}{d s}\left(\int_{\mathbb{R}^{2 N}}(x \cdot v) Q_{b}\right)=-b_{s} \int_{\mathbb{R}^{2 N}}(x \cdot v) \frac{\partial Q_{b}}{\partial b} \\
=-b_{s}\left(\int_{\mathbb{R}^{2 N}}|v|^{4-N}(x \cdot v) x \cdot \nabla_{v} Q+o(1)\right)=b_{s}\left(m^{*}+o(1)\right) .
\end{gathered}
$$


Inserting (3.30) into (3.28) completes the proof of (3.21) for $N=4$.

Case $N=3$.

We argue similarly but taking great care of the terms which are not scaling invariant. We compute from (1.3):

$$
\begin{aligned}
-\frac{d}{d t} & \left(\int_{\mathbb{R}^{2 N}}(x \cdot v) \psi f\right) \\
= & -\int_{\mathbb{R}^{2 N}} f \psi\left(\frac{|v|^{2}}{\left.\sqrt{1+|v|^{2}}-x \cdot E_{f}\right)}\right. \\
& -\int_{\mathbb{R}^{2 N}}(x \cdot v) f\left(\frac{v}{\sqrt{1+|v|^{2}}} \cdot \nabla_{x} \psi-E_{f} \cdot \nabla_{v} \psi\right) .
\end{aligned}
$$

We now inject the conservation of the Hamiltonian

$$
\mathcal{H}\left(f_{0}\right)=\int_{\mathbb{R}^{2 N}} \sqrt{1+|v|^{2}} f-\frac{1}{2} \int\left|E_{f}\right|^{2}=\int_{\mathbb{R}^{2 N}} \sqrt{1+|v|^{2}} f-\int_{\mathbb{R}^{2 N}} f x \cdot E_{f}
$$

to get

$$
\begin{aligned}
-\frac{d}{d t}\left(\int_{\mathbb{R}^{2 N}}(x \cdot v) \psi f\right)= & \int_{\mathbb{R}^{2 N}}(1-\psi) f\left(\sqrt{1+|v|^{2}}-x \cdot E_{f}\right) \\
& +\int_{\mathbb{R}^{2 N}} \psi f\left(\sqrt{1+|v|^{2}}-\frac{|v|^{2}}{\sqrt{1+|v|^{2}}}\right) \\
& -\mathcal{H}\left(f_{0}\right)-\int_{\mathbb{R}^{2 N}}(x \cdot v) f\left(\frac{v}{\sqrt{1+|v|^{2}}} \cdot \nabla_{x} \psi-E_{f} \cdot \nabla_{v} \psi\right) .
\end{aligned}
$$

We now rescale according to (3.24) and (3.2) and obtain:

$$
\begin{aligned}
-\frac{d}{d s}( & \left.\int_{\mathbb{R}^{2 N}}(x \cdot v) \psi g\right) \\
= & \int_{\mathbb{R}^{2 N}}(1-\psi) g\left(|v|-x \cdot E_{g}\right)-\lambda \mathcal{H}\left(f_{0}\right) \\
& +\int_{\mathbb{R}^{2 N}} \psi g\left(\sqrt{\lambda^{2}+|v|^{2}}-\frac{|v|^{2}}{\sqrt{\lambda^{2}+|v|^{2}}}\right) \\
& -\int_{\mathbb{R}^{2 N}}(x \cdot v) g\left(\frac{v}{|v|} \cdot \nabla_{x} \psi-E_{g} \cdot \nabla_{v} \psi\right) \\
& -\int_{\mathbb{R}^{2 N}}(x \cdot v) g\left(\frac{v}{\sqrt{\lambda^{2}+|v|^{2}}}-\frac{v}{|v|}\right) \cdot \nabla_{x} \psi \\
\geq & \int_{\mathbb{R}^{2 N}}(1-\psi) g\left(|v|-x \cdot E_{g}\right)-\lambda \mathcal{H}\left(f_{0}\right) \\
& -\int_{\mathbb{R}^{2 N}} g(x \cdot v)\left(\frac{v}{|v|} \cdot \nabla_{x} \psi-E_{g} \cdot \nabla_{v} \psi\right) \\
& -\int_{\mathbb{R}^{2 N}}(x \cdot v) g\left(\frac{v}{\sqrt{\lambda^{2}+|v|^{2}}}-\frac{v}{|v|}\right) \cdot \nabla_{x} \psi .
\end{aligned}
$$


The first three terms are treated as in the case $N=4$. Indeed, we have as for (3.30)

$$
-\frac{d}{d s}\left(\int_{\mathbb{R}^{2 N}}(x \cdot v) \psi g\right)=b_{s}\left(m^{*}+o(1)\right)
$$

Similary as in (3.26),

$$
|v|-x \cdot E_{g}=\frac{|v|}{2}+\frac{1}{|x|}\left(\frac{|x||v|}{2}-|x|^{2} E_{g}(|x|)\right) \geq \frac{|v|}{2}+\frac{1}{|x|}\left(\frac{|x||v|}{2}-\frac{|f|_{L^{1}}}{\sigma_{3}}\right) \geq \frac{|v|}{2}
$$

for $(x, v) \in \operatorname{Supp}(\psi)$, and eventually

$$
-\int_{\mathbb{R}^{2 N}}(x \cdot v) g\left(\frac{v}{|v|} \nabla_{x} \psi-E_{g} \cdot \nabla_{v} \psi\right)=-2 \int_{\mathbb{R}^{2 N}} g \frac{(x \cdot v)^{2}}{|x||v|}\left(|v|-x \cdot E_{g}\right) \psi^{\prime}(|x||v|) \geq 0 .
$$

It remains to treat the last term in (3.31). Observe that

$$
\left|\frac{v}{\sqrt{\lambda^{2}+|v|^{2}}}-\frac{v}{|v|}\right|=\frac{\lambda^{2}}{\sqrt{\lambda^{2}+|v|^{2}}\left(\sqrt{\lambda^{2}+|v|^{2}}+|v|\right)} \leq \lambda \text { for }|v| \geq \sqrt{\lambda}
$$

and thus

$$
\left|\int_{|v| \geq \sqrt{\lambda}}(x \cdot v) g\left(\frac{v}{\sqrt{\lambda^{2}+|v|^{2}}}-\frac{v}{|v|}\right) \cdot \nabla_{x} \psi\right| \leq C \lambda \int|v| g \leq C \lambda .
$$

For the other term, we have

$$
\left|\int_{|v| \leq \sqrt{\lambda}}(x \cdot v) g\left(\frac{v}{\sqrt{\lambda^{2}+|v|^{2}}}-\frac{v}{|v|}\right) \cdot \nabla_{x} \psi\right| \leq C \int_{|v| \leq \sqrt{\lambda}} g|v| \leq C \sqrt{\lambda} .
$$

Inserting (3.32), (3.33), (3.34), (3.35) and (3.36) into (3.31) now yields (3.21) for $N=3$.

Step 2. Almost monotonicity of $b$.

We now turn to the proof of (3.22). Integrating (3.21) in time on $\left[s_{0}, s\right)$, we get

$$
b(s) \geq b(0)-C\left(1+\left|\mathcal{H}\left(f_{0}\right)\right|\right) \int_{s_{0}}^{s} \sqrt{\lambda(s)} d s
$$

To evaluate the time integral in (3.37), we use (3.13) and (3.2) which imply

$$
\int_{s_{0}}^{s_{1}} \sqrt{\lambda(s)} d s \leq \int_{s_{0}}^{+\infty} e^{-\frac{b(0)}{10} s} d s=\frac{10}{b(0)} e^{-\frac{b(0)}{10} s_{0}}=\frac{10}{b(0)} \lambda_{0}^{\frac{1}{10}}
$$


and thus from (3.10)

$$
C\left(1+\left|\mathcal{H}\left(f_{0}\right)\right|\right) \int_{s_{0}}^{s} \sqrt{\lambda(s)} d s \leq \frac{C}{b(0)} \lambda_{0}^{\frac{1}{10}}\left(1+\left|\mathcal{H}\left(f_{0}\right)\right|\right) \leq C b(0)^{2} .
$$

Injecting this into (3.37) yields

$$
b(t)(1+\delta(b(t))) \geq b(0)(1-\delta(b(0)))
$$

and (3.22) now follows from (3.14).

This concludes the proof of Lemma 4

3.3. Coercivity of the linearized energy and control of $|\varepsilon|_{\varepsilon}$ and $b$. The second major input of our analysis is, as a consequence of the conservation of the Hamiltonian and the $L^{1}, L^{p}$ norm and the almost monotonicity of $b$ (3.22), the following uniform control on $|\varepsilon|_{\varepsilon}$ and $b$ :

Lemma 5 (Control of $|\varepsilon|_{\varepsilon}$ and $b$ ). It holds that $\forall t \in\left[0, t_{1}\right)$,

$$
\begin{gathered}
\int_{\mathbb{R}^{2 N}} \kappa \varepsilon_{1}(t)+\int_{\mathbb{R}^{2 N}} \varepsilon_{1}^{p}(t)+\int_{\mathbb{R}^{2 N}} \varepsilon_{2}^{2}(t) Q_{b}^{p-2}+\int_{\mathbb{R}^{2 N}}\left|\varepsilon_{3}(t)\right|^{p}<\delta(b(0)) b^{2}(t), \\
b(t) \leq b(0)(1+\delta(b(0)))
\end{gathered}
$$

with $\delta(b(0)) \rightarrow 0$ as $b(0) \rightarrow 0$.

Proof of Lemma 5. This result is a consequence of the coercivity of the linearized energy under the orthogonality conditions (2.47) and the monotonicity of $b$ (3.22).

Step 1. Conservation of the Hamiltonian.

Let us write the conservation of the Hamiltonian using (2.34) and (3.24). For $N=3$, we have

$$
\begin{aligned}
\lambda \mathcal{H}\left(f_{0}\right) & =\int_{\mathbb{R}^{2 N}} \sqrt{\lambda^{2}+|v|^{2}} g-\frac{1}{2} \int_{\mathbb{R}^{N}}\left|E_{g}\right|^{2} \\
& =\int_{\mathbb{R}^{2 N}} \varepsilon\left(|v|+\phi_{b}\right)+\int_{\mathbb{R}^{2 N}}\left(\sqrt{\lambda^{2}+|v|^{2}}-|v|\right) g-\frac{1}{2} \int_{\mathbb{R}^{N}}\left|E_{\varepsilon}\right|^{2} .
\end{aligned}
$$

For $N=4$,

$$
\lambda^{2} \mathcal{H}\left(f_{0}\right)=\int_{\mathbb{R}^{2 N}} \varepsilon\left(\frac{|v|^{2}}{2}+\phi_{b}\right)-\frac{1}{2} \int_{\mathbb{R}^{N}}\left|E_{\varepsilon}\right|^{2} .
$$

We add to this the conservation of the $L^{1}, L^{p}$ norm and get in both cases using $\left(\sqrt{\lambda^{2}+|v|^{2}}-|v|\right) g \geq 0$ :

$$
\begin{aligned}
\lambda^{N-2} \mathcal{H}\left(f_{0}\right)+\int_{\mathbb{R}^{2 N}} f_{0}+\frac{1}{p} \int_{\mathbb{R}^{2 N}} f_{0}^{p} \geq & \int_{\mathbb{R}^{2 N}} \varepsilon\left(\frac{|v|^{N-2}}{N-2}+\phi_{b}\right)+\int_{\mathbb{R}^{2 N}}\left(Q_{b}+\varepsilon\right) \\
& +\frac{1}{p} \int_{\mathbb{R}^{2 N}}\left(Q_{b}+\varepsilon\right)^{p}-\frac{1}{2} \int_{\mathbb{R}^{N}}\left|E_{\varepsilon}\right|^{2} .
\end{aligned}
$$


Inserting the decomposition (3.8), this becomes, using (2.30),

$$
\begin{aligned}
\lambda^{N-2} \mathcal{H}\left(f_{0}\right)+\int_{\mathbb{R}^{2 N}} f_{0}+\frac{1}{p} \int_{\mathbb{R}^{2 N}} f_{0}^{p} \geq \int_{\mathbb{R}^{2 N}} Q_{b}+\frac{1}{p} \int_{\mathbb{R}^{2 N}} Q_{b}^{p} \\
\quad+\frac{1}{2}\left[(p-1) \int_{\mathbb{R}^{2 N}} Q_{b}^{p-2} \varepsilon_{2}^{2}-\int_{\mathbb{R}^{N}}\left|E_{\varepsilon_{2}}\right|^{2}\right]-\frac{1}{2}\left[\int_{\mathbb{R}^{N}}\left|E_{\varepsilon}\right|^{2}-\int_{\mathbb{R}^{N}}\left|E_{\varepsilon_{2}}\right|^{2}\right] \\
+\left[\int_{\mathbb{R}^{2 N}} \varepsilon_{1}\left(\frac{|v|^{N-2}}{N-2}+b \chi(x) x \cdot v+1+\phi_{b}\right)+\frac{1}{p} \int_{\mathbb{R}^{2 N}} \varepsilon_{1}^{p}\right] \\
\quad-b \int_{\mathbb{R}^{2 N}}\left(\chi(x) \varepsilon_{1}+\varepsilon_{2}+\varepsilon_{3}\right) x \cdot v \\
+\frac{1}{p}\left[\int_{\operatorname{Supp}\left(\varepsilon_{3}\right)}\left(Q_{b}+\varepsilon_{3}\right)^{p}-\int_{\operatorname{Supp}\left(\varepsilon_{3}\right)} Q_{b}^{p}-p \int_{\mathbb{R}^{2 N}} Q_{b}^{p-1} \varepsilon_{3}\right] \\
+\frac{1}{p}\left[\int_{\operatorname{Supp}\left(\varepsilon_{2}\right)}\left(Q_{b}+\varepsilon_{2}\right)^{p}-\int_{\operatorname{Supp}\left(\varepsilon_{2}\right)} Q_{b}^{p}-p \int_{\mathbb{R}^{2 N}} Q_{b}^{p-1} \varepsilon_{2}^{2}\right. \\
\left.-\frac{p(p-1)}{2} \int_{\mathbb{R}^{2 N}} Q_{b}^{p-2} \varepsilon_{2}^{2}\right] .
\end{aligned}
$$

We now claim that the right-hand side of the above equality controls $b^{2}$ and the norm (3.15). To wit, we estimate each term.

Step 2. $\varepsilon_{1}$ terms.

Let $\kappa$ be given by (3.5) and

$$
J=\left\{(x, v) \in \mathbb{R}^{2 N} \text { with } \kappa(x, v) \leq \frac{|v|^{N-2}+1}{4}\right\} ;
$$

then $J$ is compact in $(x, v)$ from (2.5) and (2.6). We now split

$$
\varepsilon_{1}=\varepsilon_{1} \mathbf{1}_{(x, v) \in J}+\varepsilon_{1} \mathbf{1}_{(x, v) \notin J}=\varepsilon_{4}+\varepsilon_{5} .
$$

Observe by definition that

$$
\int_{\mathbb{R}^{2 N}}\left(1+|v|^{N-2}\right) \varepsilon_{5} \leq C \int_{\mathbb{R}^{2 N}} \kappa \varepsilon_{1} .
$$

We moreover claim

$$
\left|\rho_{\varepsilon_{4}}\right|_{L^{4 / 3}}^{2} \leq \delta(b(0))\left(\int_{\mathbb{R}^{2 N}} \kappa \varepsilon_{1}+\int_{\mathbb{R}^{2 N}} \varepsilon_{1}^{p}\right) .
$$

Assuming (3.46), we estimate $\left|E_{\varepsilon_{1}}\right|_{L^{2}}$ :

$$
\begin{aligned}
\int_{\mathbb{R}^{N}}\left|E_{\varepsilon_{1}}\right|^{2} & \leq \int_{\mathbb{R}^{N}}\left|E_{\varepsilon_{4}}\right|^{2}+\int_{\mathbb{R}^{N}}\left|E_{\varepsilon_{5}}\right|^{2} \\
& \leq C\left|\rho_{\varepsilon_{4}}\right|_{L^{\frac{2 N}{N+2}}}^{2}+\left.\left.C|| v\right|^{N-2} \varepsilon_{5}\right|_{L^{1}}\left|\varepsilon_{5}\right|_{L^{1}}^{\frac{2 p-N}{N(p-1)}}\left|\varepsilon_{5}\right|_{L^{p}}^{\frac{p(N-2)}{N(p-1)}} \\
& \leq \delta(b(0))\left(\int_{\mathbb{R}^{2 N}} \kappa \varepsilon_{1}+\int_{\mathbb{R}^{2 N}} \varepsilon_{1}^{p}\right)
\end{aligned}
$$

where we used the fact that $\rho_{\varepsilon_{4}}$ is compactly supported from (3.43), $\frac{2 N}{N+2} \leq \frac{4}{3}$ and (3.46), and the Gagliardo-Nirenberg interpolation estimate (1.10) and (3.11). 
Proof of (3.46). Observe that $\varepsilon_{4}$ is compactly supported in $(x, v)$. Moreover, we have from direct verification

$$
\left|\int_{(x, v) \in J} \frac{d v}{\kappa^{q}(x, v)}\right|_{L_{x}^{\infty}} \leq C(q) \text { for all } 0<q<1 .
$$

Taking $q=1-\frac{1}{p}$, we get from Hölder:

$$
\rho_{\varepsilon_{4}}(x) \leq C\left|\varepsilon_{1} \kappa(x, \cdot)\right|_{L_{v}^{1}}^{\frac{(p-1)^{2}}{2 p^{2}-2 p+1}}\left|\varepsilon_{1}(x, \cdot)\right|_{L_{v}^{p}}^{\frac{p^{2}}{2 p^{2}-2 p+1}}\left|\kappa^{-q}(x, \cdot)\right|_{L_{v}^{1}}^{\frac{p(p-1)}{2 p^{2}-2 p+1}} \mathbf{1}_{|x| \leq C} .
$$

We now observe that

$$
\frac{4}{3}\left[\frac{(p-1)^{2}}{2 p^{2}-2 p+1}+\frac{p}{2 p^{2}-2 p+1}\right]<1 \text { for } p>2
$$

and thus from the Young inequality:

$$
\left|\rho_{\varepsilon_{4}}\right|_{L^{4 / 3}\left(\mathbb{R}^{N}\right)} \leq C\left|\varepsilon_{1} \kappa\right|_{L^{1}}^{\frac{(p-1)^{2}}{2 p^{2}-2 p+1}}\left|\varepsilon_{1}\right|_{L^{p}}^{\frac{p^{2}}{2 p^{2}-2 p+1}}
$$

We now square this and use Young again. Note that the dual exponent to $\alpha$ such that $\alpha \frac{2 p^{2}}{2 p^{2}-2 p+1}=p$ is $\beta=\frac{\alpha}{\alpha-1}=\frac{2 p^{2}-2 p+1}{2 p^{2}-4 p+1}$ so that

$$
\beta \frac{2(p-1)^{2}}{2 p^{2}-2 p+1}=\frac{(p-1)^{2}}{(p-1)^{2}-\frac{1}{2}}>1
$$

and (3.46) follows from Hölder again and (3.11).

Step 3. $\varepsilon_{3}$ terms.

The definition (3.8) of $\varepsilon_{3}$ and the constraint $g \geq 0$ imply

$$
\left|\varepsilon_{3}(x)\right| \geq \eta Q_{b}(x) \text { and } \frac{\varepsilon_{3}(x)}{Q_{b}(x)} \geq-1
$$

and thus (3.7) yields

$$
\int_{\operatorname{Supp}\left(\varepsilon_{3}\right)}\left|Q_{b}+\varepsilon_{3}\right|^{p}-\int_{\operatorname{Supp}\left(\varepsilon_{3}\right)} Q_{b}^{p}-p \int_{\mathbb{R}^{2 N}} Q_{b}^{p-1} \varepsilon_{3} \geq C \int_{\mathbb{R}^{2 N}}\left|\varepsilon_{3}\right|^{p} .
$$

On the other hand, observe from (2.30) that

$$
\left|\int_{v \in \mathbb{R}^{N}} \frac{d v}{\left[Q_{b}^{p-1}(x, v)\right]^{1-\frac{1}{p}}}\right|_{L_{x}^{\infty}} \leq C
$$


and we thus get arguing as for the proof of (3.46),

$$
\begin{aligned}
\left|\rho_{\left|\varepsilon_{3}\right|}\right|_{L^{4 / 3}}^{2}+\int_{\mathbb{R}^{N}}\left|E_{\varepsilon_{3}}\right|^{2} & \leq \delta(b(0))\left(\int_{\mathbb{R}^{2 N}} Q_{b}^{p-1}\left|\varepsilon_{3}\right|+\int_{\mathbb{R}^{2 N}}\left|\varepsilon_{3}\right|^{p}\right) \\
& \leq \delta(b(0)) \int_{\mathbb{R}^{2 N}}\left|\varepsilon_{3}\right|^{p}
\end{aligned}
$$

where we used (3.48) again. The $\varepsilon_{1}, \varepsilon_{2}, \varepsilon_{3}$ term in (3.42) is estimated using the orthogonality condition (2.47), (2.42), the decomposition (3.44) and the fact that $|x||v| \leq C$ on $\operatorname{Supp}(\psi)$ from (2.39):

$$
\begin{aligned}
& \left|b \int_{\mathbb{R}^{2 N}}\left(\chi(x) \varepsilon_{1}+\varepsilon_{2}+\varepsilon_{3}\right) x \cdot v\right| \\
& \leq|b| \int_{\mathbb{R}^{2 N}} \varepsilon_{1}(\chi(x)+\psi(x))|x||v| \leq C|b|\left(\int_{\mathbb{R}^{2 N}} \varepsilon_{4}+\varepsilon_{5}\right) \\
& \quad \leq C|b|\left(\left|\rho_{\varepsilon_{4}}\right|_{L^{4 / 3}}+\int \varepsilon_{5}\right) \leq \delta(b(0))\left\{b^{2}+\int_{\mathbb{R}^{2 N}} \kappa \varepsilon_{1}+\int \varepsilon_{1}^{p}\right\}
\end{aligned}
$$

with $\delta(b(0)) \rightarrow 0$ as $b(0) \rightarrow 0$ from (3.45) and (3.46) .

Step 4. Coercivity of the linearized energy.

The main input is the coercivity of the linearized energy which is a consequence of the variational characterization of $Q$. The following proposition has been proved in [27].

Proposition 5 (Coercivity of the linearized energy, 27). Let $N=3,4,2<p<$ $\infty)$; then there exists a universal constant $\delta_{0}=\delta_{0}(N, p)>0$ such that for all $h$ with radial symmetry and compactly supported in $\operatorname{Supp}(Q)$, it holds that

$$
\begin{aligned}
& (p-1) \int_{\mathbb{R}^{2 N}} Q^{p-2} h^{2}-\int_{\mathbb{R}^{N}}\left|E_{h}\right|^{2} \\
& \quad \geq \delta_{0} \int_{\mathbb{R}^{2 N}} Q^{p-2} h^{2}-\frac{1}{\delta_{0}}\left\{\left(\int_{\mathbb{R}^{2 N}} h|v|^{4-N}|x|^{2}\right)^{2}+\left(\int_{\mathbb{R}^{2 N}} h\left(\frac{|v|^{N-2}}{N-2}+\phi_{Q}\right)\right)^{2}\right\} .
\end{aligned}
$$

Observe from (3.8) that $\varepsilon_{2}$ is supported on $K_{b}$ and not on $\operatorname{Supp}(Q)$ and that the quadratic form appearing in (3.42) involves $Q_{b}$ and not $Q$. We will therefore need the following coercivity property: there exists $b^{*}>0$ small enough such that for all $|b|<b^{*}$, for all $h$ with $\operatorname{Supp}(h) \in K_{b}=\operatorname{Supp}\left(Q_{b}\right)$, it holds that

$$
\begin{aligned}
(p-1) & \int_{\mathbb{R}^{2 N}} Q_{b}^{p-2} h^{2}-\int_{\mathbb{R}^{N}}\left|E_{h}\right|^{2} \\
& \geq \frac{\delta_{0}}{2} \int_{\mathbb{R}^{2 N}} Q_{b}^{p-2} h^{2}-\frac{2}{\delta_{0}}\left\{\left(\int_{\mathbb{R}^{2 N}} h|v|^{4-N}|x|^{2}\right)^{2}+\left(\int_{\mathbb{R}^{2 N}} h\left(\frac{|v|^{N-2}}{N-2}+\phi_{b}\right)\right)^{2}\right\} .
\end{aligned}
$$

Proof of (3.53). This is a simple consequence of (3.52). Arguing by contradiction, we assume the existence of two sequences $b_{n} \rightarrow 0, h_{n}$ with radial symmetry and supported in $K_{b_{n}}$, such that

$$
\int_{\mathbb{R}^{2 N}} Q_{b_{n}}^{p-2} h_{n}^{2}=1
$$


and such that (3.53) does not hold, i.e.

$$
\begin{aligned}
\int_{\mathbb{R}^{N}}\left|E_{h_{n}}\right|^{2}>(p-1)-\frac{\delta_{0}}{2}+\frac{2}{\delta_{0}}\{ & \left(\int_{\mathbb{R}^{2 N}} h_{n}|v|^{4-N}|x|^{2}\right)^{2} \\
& \left.+\left(\int_{\mathbb{R}^{2 N}} h_{n}\left(\frac{|v|^{N-2}}{N-2}+\phi_{b_{n}}\right)\right)^{2}\right\} .
\end{aligned}
$$

Observing from the $Q_{b}$ equation (2.30) and from direct computation that, for $b$ small enough,

$$
\forall \theta<\frac{N(p-1)}{N-2}, \quad\left|\int_{\mathbb{R}^{N}} \frac{d v}{Q_{b}^{\theta}(x, v)}\right|_{L_{x}^{\infty}} \leq C,
$$

we deduce from the Hölder inequality that for every function $h$ supported on $K_{b}$ we have

$$
|h|_{L^{q}} \leq C_{q}\left(\int_{\mathbb{R}^{2 N}} Q_{b}^{p-2} h^{2}\right)^{1 / 2} \quad \forall q<\frac{2 N(p-1)}{N(p-1)+(N-2)(p-2)} .
$$

Hence, from (3.54), (3.57) and the uniform convergence $Q_{b_{n}} \rightarrow Q$ as $n \rightarrow \infty$ deduced from (2.32), we get for some $q>1$, up to a subsequence,

$$
h_{n} \rightarrow h \quad \text { in } L^{q} \quad \text { and } \quad Q_{b_{n}}^{\frac{p-2}{2}} h_{n} \rightarrow Q^{\frac{p-2}{2}} h \quad \text { in } L^{2},
$$

and by lower semicontinuity

$$
\int Q^{p-2} h^{2} \leq 1
$$

Moreover, from (3.56) with $\theta=p-2$ and Hölder, we get for all $h$ supported on $K_{b}$

$$
\left|\rho_{|h|}\right|_{L^{2}} \leq C\left(\int_{\mathbb{R}^{2 N}} Q_{b}^{p-2} h^{2}\right)^{1 / 2}
$$

From a standard compactness result, $\rho_{\left|h_{n}\right|}$ being radially symmetric and since $2>$ $\frac{2 N}{N+2}$, we deduce from (3.54) that the sequence $E_{h_{n}}$ is compact in $L^{2}$. Passing to the limit in (3.55) thus yields

$$
\int_{\mathbb{R}^{N}}\left|E_{h}\right|^{2} \geq(p-1)-\frac{\delta_{0}}{2}+\frac{2}{\delta_{0}}\left\{\left(\int_{\mathbb{R}^{2 N}} h|v|^{4-N}|x|^{2}\right)^{2}+\left(\int_{\mathbb{R}^{2 N}} h\left(\frac{|v|^{N-2}}{N-2}+\phi_{Q}\right)\right)^{2}\right\} .
$$

A first consequence of (3.60) is that $f \neq 0$ (note from (3.52) that $0<\delta_{0} \leq p-1$ ). A second consequence of (3.60), together with (3.58), is

$$
\begin{aligned}
\int_{\mathbb{R}^{N}}\left|E_{h}\right|^{2} \geq( & -1) \int_{\mathbb{R}^{2 N}} Q^{p-2} h^{2}-\frac{\delta_{0}}{2} \int_{\mathbb{R}^{2 N}} Q^{p-2} h^{2} \\
& +\frac{2}{\delta_{0}}\left\{\left(\int_{\mathbb{R}^{2 N}} h|v|^{4-N}|x|^{2}\right)^{2}+\left(\int_{\mathbb{R}^{2 N}} h\left(\frac{|v|^{N-2}}{N-2}+\phi_{Q}\right)\right)^{2}\right\}
\end{aligned}
$$

which, since $h \neq 0$, contradicts (3.52). The proof of the claim (3.53) is complete. 
1052

MOHAMMED LEMOU, FLORIAN MÉHATS, AND PIERRE RAPHAËL

Step 5. Control of the $\varepsilon_{2}$ terms.

We now claim that (3.53) implies

$$
\begin{aligned}
(p-1) \int_{\mathbb{R}^{2 N}} Q_{b}^{p-2} \varepsilon_{2}^{2}-\int\left|E_{\varepsilon_{2}}\right|^{2} \geq & \frac{\delta_{0}}{10} \int_{\mathbb{R}^{2 N}} Q_{b}^{p-2} \varepsilon_{2}^{2} \\
& -\delta(b(0))\left\{b^{2}+\int_{\mathbb{R}^{2 N}}\left|\varepsilon_{3}\right|^{p}+\int_{\mathbb{R}^{2 N}} \kappa \varepsilon_{1}+\int \varepsilon_{1}^{p}\right\} .
\end{aligned}
$$

Proof of (3.61). Applying (3.53) to $\varepsilon_{2}$, we need to estimate the negative inner products in the right-hand side of (3.53).

The first inner product term is controlled using the orthogonality condition (2.47), (3.46) and (3.50) which implies

$(3.62)$

$$
\begin{gathered}
\left\{\int_{\mathbb{R}^{2 N}} \varepsilon_{2}|v|^{4-N}|x|^{2}\right\}^{2} \\
\leq C\left\{\left(\int_{\mathbb{R}^{2 N}} \varepsilon|v|^{4-N}|x|^{2} \zeta\right)^{2}+\left(\int_{\mathbb{R}^{2 N}} \varepsilon_{1}|v|^{4-N}|x|^{2} \zeta\right)^{2}+\left(\int_{\mathbb{R}^{2 N}} \varepsilon_{3}|v|^{4-N}|x|^{2} \zeta\right)^{2}\right\} \\
\leq C\left\{\left|\rho_{\left|\varepsilon_{3}\right|}\right|_{L^{4 / 3}}+\left|\rho_{\varepsilon_{4}}\right|_{L^{4 / 3}}+\int_{\mathbb{R}^{2 N}} \kappa \varepsilon_{1}\right\}^{2} \\
\leq \delta(b(0))\left\{\int_{\mathbb{R}^{2 N}}\left|\varepsilon_{3}\right|^{p}+\int_{\mathbb{R}^{2 N}} \kappa \varepsilon_{1}+\int_{\mathbb{R}^{2 N}} \varepsilon_{1}^{p}\right\} .
\end{gathered}
$$

The control of the second inner product follows from the conservation of the Hailtonian and (3.46). Indeed,

$$
\begin{aligned}
\left\{\int_{\mathbb{R}^{2 N}} \varepsilon_{2} \frac{|v|^{N-2}}{N-2}+\phi_{b}\right\}^{2} & \\
\leq C\left\{\left(\int_{\mathbb{R}^{2 N}} \varepsilon\left(\frac{|v|^{N-2}}{N-2}+\phi_{b}\right)\right)^{2}\right. & +\left(\int_{\mathbb{R}^{2 N}} \varepsilon_{1}\left(\frac{|v|^{N-2}}{N-2}+\phi_{b}\right)\right)^{2} \\
& \left.+\left(\int_{\mathbb{R}^{2 N}} \varepsilon_{3}\left(\frac{|v|^{N-2}}{N-2}+\phi_{b}\right)\right)^{2}\right\} .
\end{aligned}
$$

For the third term in (3.63), we have from (3.50)

$$
\left\{\int_{\mathbb{R}^{2 N}} \varepsilon_{3}\left(\frac{|v|^{N-2}}{2}+\phi_{b}\right)\right\}^{2} \leq C\left|\rho_{\left|\varepsilon_{3}\right|}\right|_{L^{4 / 3}}^{2} \leq \delta(b(0)) \int_{\mathbb{R}^{2 N}}\left|\varepsilon_{3}\right|^{p} .
$$

The second term in (3.63) is estimated using the decomposition (3.44). Indeed, observe from (3.43) and (3.11) that

$$
\left\{\int_{\mathbb{R}^{2 N}} \varepsilon_{5}\left(\frac{|v|^{N-2}}{N-2}+\phi_{b}\right)\right\}^{2} \leq \delta(b(0)) \int_{\mathbb{R}^{2 N}} \kappa \varepsilon_{1} .
$$


To estimate the $\varepsilon_{4}$ term, we use the fact that $\varepsilon_{4}$ is compactly supported, Hölder and (3.46), (3.11):

$$
\left\{\int_{\mathbb{R}^{2 N}} \varepsilon_{4}\left(\frac{|v|^{N-2}}{N-2}+\phi_{b}\right)\right\}^{2} \leq C\left|\rho_{\varepsilon_{4}}\right|_{L^{4 / 3}}^{2} \leq \delta(b(0))\left\{\int_{\mathbb{R}^{2 N}} \kappa \varepsilon_{1}+\int_{\mathbb{R}^{2 N}} \varepsilon_{1}^{p}\right\} .
$$

It remains to estimate the first term in (3.63) which is now controlled from the conservation of the Hamiltonian, and we claim

$$
\begin{aligned}
& \left\{\int_{\mathbb{R}^{2 N}} \varepsilon\left(\frac{|v|^{N-2}}{N-2}+\phi_{b}\right)\right\}^{2} \\
& \quad \leq \delta(b(0))\left\{b^{2}+\int_{\mathbb{R}^{2 N}}\left|\varepsilon_{3}\right|^{p}+\int_{\mathbb{R}^{2 N}} \kappa \varepsilon_{1}+\int_{\mathbb{R}^{2 N}} \varepsilon_{1}^{p}+\int_{\mathbb{R}^{2 N}} Q_{b}^{p-2} \varepsilon_{2}^{2}\right\} .
\end{aligned}
$$

Inserting (3.64), (3.65), (3.66) and the claim (3.67) into (3.63) yields

$$
\begin{aligned}
& \left\{\int_{\mathbb{R}^{2 N}} \varepsilon_{2}\left(\frac{|v|^{N-2}}{N-2}+\phi_{b}\right)\right\}^{2} \\
& \quad \leq \delta(b(0))\left\{b^{2}+\int_{\mathbb{R}^{2 N}}\left|\varepsilon_{3}\right|^{p}+\int_{\mathbb{R}^{2 N}} \kappa \varepsilon_{1}+\int_{\mathbb{R}^{2 N}} \varepsilon_{1}^{p}+\int_{\mathbb{R}^{2 N}} Q_{b}^{p-2} \varepsilon_{2}^{2}\right\}
\end{aligned}
$$

which together with (3.62) and (3.53) concludes the proof of (3.61).

Proof of (3.67). First remark from (3.10), (3.12) and (3.14) that

$$
\lambda^{N-2}(t)\left(1+\left|\mathcal{H}\left(f_{0}\right)\right|\right)<b^{3}(0)
$$

and thus (3.40) and (3.41) imply

$$
\begin{aligned}
& \left\{\int_{\mathbb{R}^{2 N}} \varepsilon\left(\frac{|v|^{N-2}}{2}+\phi_{b}\right)\right\}^{2} \leq C\left(\lambda^{N-2} \mathcal{H}\left(f_{0}\right)\right)^{2}+\left|E_{\varepsilon}\right|_{L^{2}}^{4}+\left(\int_{\mathbb{R}^{2 N}}\left(\sqrt{\lambda^{2}+|v|^{2}}-|v|\right) g\right)^{2} \\
& \leq C\left\{b^{3}(0)+\left|E_{\varepsilon}\right|_{L^{2}}^{4}+\left(\int_{\mathbb{R}^{2 N}}\left(\sqrt{\lambda^{2}+|v|^{2}}-|v|\right) g\right)^{2}\right\}
\end{aligned}
$$

where the last term is there only for $N=3$. To estimate the field term, first recall the estimate

$$
\int_{\mathbb{R}^{N}}\left|E_{\varepsilon_{2}}\right|^{2} \leq C \int_{\mathbb{R}^{2 N}} Q_{b}^{p-2} \varepsilon_{2}^{2}
$$

obtained from (3.59). From (3.70), (3.47), (3.50), we get

$$
\left|E_{\varepsilon}\right|_{L^{2}}^{4} \leq \delta(b(0))\left\{\int_{\mathbb{R}^{2 N}}\left|\varepsilon_{3}\right|^{p}+\int_{\mathbb{R}^{2 N}} \kappa \varepsilon_{1}+\int_{\mathbb{R}^{2 N}} \varepsilon_{1}^{p}+\int_{\mathbb{R}^{2 N}} Q_{b}^{p-2} \varepsilon_{2}^{2}\right\} .
$$

Inserting (3.71) into (3.69) concludes the proof of (3.67) for $N=4$. For $N=3$, it remains to treat the last term in (3.69). Remark that $0<\sqrt{\lambda^{2}+|v|^{2}}-|v|=$ $\frac{\lambda^{2}}{\sqrt{\lambda^{2}+|v|^{2}}+|v|} \leq \lambda$ and thus

$$
\left(\int_{\mathbb{R}^{2 N}}\left(\sqrt{\lambda^{2}+|v|^{2}}-|v|\right) g\right)^{2} \leq C \lambda^{2} \leq \delta(b(0)) b^{2}
$$

from (3.10) and (3.12). This together with (3.69) and (3.71) concludes the proof of (3.67) for $N=3$. 
Finally, we estimate the nonlinear term in $\varepsilon_{2}$ in (3.42) using the definition (3.8) of $\varepsilon_{2}$ and (3.6):

$$
\begin{aligned}
& \left|\int_{\operatorname{Supp}\left(\varepsilon_{2}\right)}\left(Q_{b}+\varepsilon_{2}\right)^{p}-\int_{\operatorname{Supp}\left(\varepsilon_{2}\right)} Q_{b}^{p}-p \int_{\mathbb{R}^{2 N}} Q_{b}^{p-1} \varepsilon_{2}-\frac{p(p-1)}{2} \int_{\mathbb{R}^{2 N}} Q_{b}^{p-2} \varepsilon_{2}^{2}\right| \\
& \quad \leq \frac{\delta_{0}}{100} \int Q_{b}^{p-2} \varepsilon_{2}^{2} .
\end{aligned}
$$

Step 6. Conclusion.

We now insert (3.46), (3.61), (3.68), (3.72), (3.49), (3.50) and (3.51) into (3.42) to derive so far

$$
\begin{aligned}
& b^{2} \delta(b(0))+\int_{\mathbb{R}^{2 N}} f_{0}+\frac{1}{p} \int_{\mathbb{R}^{2 N}} f_{0}^{p} \geq \int_{\mathbb{R}^{2 N}} Q_{b}+\frac{1}{p} \int_{\mathbb{R}^{2 N}} Q_{b}^{p} \\
+ & \delta_{1}\left\{\int_{\mathbb{R}^{2 N}} \kappa \varepsilon_{1}+\int_{\mathbb{R}^{2 N}} \varepsilon_{1}^{p}+\int_{\mathbb{R}^{2 N}}\left|\varepsilon_{3}\right|^{p}+\int_{\mathbb{R}^{2 N}} Q_{b}^{p-2} \varepsilon_{2}^{2}\right\}
\end{aligned}
$$

for some universal constant $\delta_{1}>0$. Observe from (3.9) that

$$
\int_{\mathbb{R}^{2 N}} f_{0}+\frac{1}{p} \int_{\mathbb{R}^{2 N}} f_{0}^{p} \leq \int_{\mathbb{R}^{2 N}} Q_{b(0)}+\frac{1}{p} \int_{\mathbb{R}^{2 N}} Q_{b(0)}^{p}+C b^{3}(0)
$$

and thus (3.14) and the supercriticality of the $Q_{b}$ mass (2.35) imply

$$
\begin{aligned}
& b^{2}(t) \delta(b(0))+m^{*} b^{2}(0) \geq m^{*} b^{2}(t) \\
+ & \delta_{1}\left\{\int_{\mathbb{R}^{2 N}} \kappa \varepsilon_{1}+\int_{\mathbb{R}^{2 N}} \varepsilon_{1}^{p}+\int_{\mathbb{R}^{2 N}}\left|\varepsilon_{3}\right|^{p}+\int_{\mathbb{R}^{2 N}} Q_{b}^{p-2} \varepsilon_{2}^{2}\right\} .
\end{aligned}
$$

Now injecting the monotonicity (3.22) yields (3.38) and (3.39).

This concludes the proof of Lemma 5 .

3.4. Proof of Lemma 3, Let us observe that estimates (3.22), (3.38) and (3.39) imply (3.15) and (3.17). In order to conclude the proof of Lemma 3, it thus remains to prove (3.16) and (3.18). These are a consequence of the modulation equation governing the geometrical parameters $\lambda$ and $b$ which follows from our choice of orthogonality conditions (2.47).

Proof of Lemma 3. Step 1. Pointwise in time control of $\varepsilon$.

We first claim as a consequence of (3.38) the following pointwise control on $\varepsilon$ :

$$
\int_{\mathbb{R}^{2 N}}\left(1+|v|^{N-2}\right)|\varepsilon|+\left|E_{\varepsilon}\right|_{L^{2}} \leq \delta(b(0)) b .
$$

Indeed, recall from (3.8) and (3.44) that $\varepsilon=\varepsilon_{1}+\varepsilon_{2}+\varepsilon_{3}=\varepsilon_{4}+\varepsilon_{5}+\varepsilon_{2}+\varepsilon_{3}$ where only $\varepsilon_{5}$ is not compactly supported. From their definition, we then estimate

$$
\begin{aligned}
\int_{\mathbb{R}^{2 N}} & \left(1+|v|^{N-2}\right)|\varepsilon| \\
\leq & C\left[\int_{\mathbb{R}^{2 N}}\left(1+|v|^{N-2}\right) \varepsilon_{5}+\left(\int_{\mathbb{R}^{2 N}} \varepsilon_{2}^{2} Q_{b}^{p-2}\right)^{\frac{1}{2}}+\left|\rho_{\left|\varepsilon_{3}\right|}\right|_{L^{4 / 3}}+\left|\rho_{\varepsilon_{4}}\right|_{L^{4 / 3}}\right] \\
\leq & C\left[\int_{\mathbb{R}^{2 N}} \kappa \varepsilon_{1}+\int_{\mathbb{R}^{2 N}} \varepsilon_{1}^{p}+\int_{\mathbb{R}^{2 N}} \varepsilon_{2}^{2} Q_{b}^{p-2}+\int_{\mathbb{R}^{2 N}}\left|\varepsilon_{3}\right|^{p}\right]^{\frac{1}{2}} \leq \delta(b(0)) b
\end{aligned}
$$


where we used (3.38), (3.45), (3.46), (3.50) and (3.59). The control of the potential energy follows from (3.71) and (3.38).

Step 2. Pointwise control of the modulation parameters.

We now claim the following control on the modulation parameters:

$$
\begin{gathered}
\left|b_{s}\right| \leq b \delta(b(0)) \\
\left|\frac{\lambda_{s}}{\lambda}+b\right| \leq b \delta(b(0)) .
\end{gathered}
$$

Proof of (3.74). For the sake of clarity, we distinguish the cases $N=3$ and $N=4$.

Case $N=4$ : Let us recall (3.25) and (3.30) which imply

$$
\begin{aligned}
b_{s}\left(m^{*}+o(1)\right) & =\int(1-\psi) g\left(|v|^{2}-x \cdot E_{g}\right)-2 \lambda^{2} \mathcal{H}\left(f_{0}\right) \\
& -\int_{\mathbb{R}^{2 N}} g(x \cdot v)\left(v \cdot \nabla_{x} \psi-E_{g} \cdot \nabla_{v} \psi\right) .
\end{aligned}
$$

Moreover, recall from (2.42) and (2.32) that $g=\varepsilon$ on $\operatorname{Supp}(1-\psi)$. From (2.39), $|x||v| \geq C$ on $\operatorname{Supp}(1-\psi)$ and we thus estimate using (2.38):

$$
\begin{aligned}
\left|b_{s}\right| & \leq C \int_{\mathbb{R}^{2 N}}|\varepsilon|\left(1+|v|^{2}+\frac{|f|_{L^{1}}}{|x|^{2}}\right)+C \lambda^{2}\left|\mathcal{H}\left(f_{0}\right)\right| \\
& \leq C\left[\int|\varepsilon|\left(1+|v|^{2}\right)+\lambda^{2}\left|\mathcal{H}\left(f_{0}\right)\right|\right] \leq \delta(b(0)) b
\end{aligned}
$$

from (3.73), (3.10), (3.12) and (3.14). This is (3.74) for $N=4$.

Case $N=3$ : Similarly, we have from (3.31) and 3.30):

$$
\begin{aligned}
b_{s}\left(m^{*}+o(1)\right)= & \int_{\mathbb{R}^{2 N}}(1-\psi) g\left(|v|-x \cdot E_{g}\right)-\lambda \mathcal{H}\left(f_{0}\right) \\
& +\int_{\mathbb{R}^{2 N}} \psi g\left(\sqrt{\lambda^{2}+|v|^{2}}-\frac{|v|^{2}}{\sqrt{\lambda^{2}+|v|^{2}}}\right) \\
& -\int_{\mathbb{R}^{2 N}} g(x \cdot v)\left(\frac{v}{|v|} \cdot \nabla_{x} \psi-E_{g} \cdot \nabla_{v} \psi\right) \\
& -\int_{\mathbb{R}^{2 N}} g(x \cdot v)\left(\frac{v}{\sqrt{\lambda^{2}+|v|^{2}}}-\frac{v}{|v|}\right) \cdot \nabla_{x} \psi .
\end{aligned}
$$

Using $\left|\sqrt{\lambda^{2}+|v|^{2}}-\frac{|v|^{2}}{\sqrt{\lambda^{2}+|v|^{2}}}\right|=\frac{\lambda^{2}}{\sqrt{\lambda^{2}+|v|^{2}}} \leq C \lambda$, we estimate again from (2.38)

$$
\begin{aligned}
\left|b_{s}\right| & \leq C\left[\int_{\mathbb{R}^{2 N}}|\varepsilon|\left(1+|v|+\frac{|f|_{L^{1}}}{|x|}\right)+\lambda\left(1+\left|\mathcal{H}\left(f_{0}\right)\right|\right)\right] \\
& \leq C\left[\int_{\mathbb{R}^{2 N}}|\varepsilon|(1+|v|)+\lambda\left(1+\left|\mathcal{H}\left(f_{0}\right)\right|\right)\right] \\
& \leq \delta\left(b_{0}\right) b_{0}
\end{aligned}
$$

from (3.73), 3.10) and (3.12). This is (3.74) for $N=3$. 
Proof of (3.75). Again, we distinguish the cases $N=3$ and $N=4$.

Case $N=4$ : Let us write the equation for $g$ :

$$
\partial_{s} g+v \cdot \nabla_{x} g-E_{g} \cdot \nabla_{v} g=\frac{\lambda_{s}}{\lambda}\left(x \cdot \nabla_{x} g-v \cdot \nabla_{v} g\right)
$$

Expanding $g=Q_{b}+\varepsilon$ and using (1.21) yields

$$
\begin{aligned}
& b_{s} \frac{\partial Q_{b}}{\partial b}+\partial_{s} \varepsilon+v \cdot \nabla_{x} \varepsilon-E_{Q_{b}} \cdot \nabla_{v} \varepsilon-E_{\varepsilon} \cdot \nabla_{v} Q_{b}+b\left(x \cdot \nabla_{x} \varepsilon-v \cdot \nabla_{v} \varepsilon\right) \\
& \quad=\left(\frac{\lambda_{s}}{\lambda}+b\right)\left(x \cdot \nabla_{x} Q_{b}-v \cdot \nabla_{v} Q_{b}\right)+\left(\frac{\lambda_{s}}{\lambda}+b\right)\left(x \cdot \nabla_{x} \varepsilon-v \cdot \nabla_{v} \varepsilon\right)+E_{\varepsilon} \cdot \nabla_{v} \varepsilon .
\end{aligned}
$$

We now take the inner product of (3.76) with $|x|^{2} \zeta$, use (2.47) and integrate by parts to derive

$$
\begin{aligned}
& \left(\frac{\lambda_{s}}{\lambda}+b\right) \int_{\mathbb{R}^{2 N}}\left(x \cdot \nabla_{x} Q_{b}-v \cdot \nabla_{v} Q_{b}\right)|x|^{2} \\
= & +\left(\frac{\lambda_{s}}{\lambda}+b\right) \int_{\mathbb{R}^{2 N}} \varepsilon\left(x \cdot \nabla_{x}\left(|x|^{2} \zeta\right)-v \cdot \nabla_{v}\left(|x|^{2} \zeta\right)\right)+b_{s} \frac{d}{d b} \int_{\mathbb{R}^{2 N}}|x|^{2} Q_{b} \\
- & 2 \int_{\mathbb{R}^{2 N}} \varepsilon(x \cdot v) \zeta-\int_{\mathbb{R}^{2 N}} \varepsilon|x|^{2}\left(v \cdot \nabla_{x} \zeta-E_{Q_{b}} \cdot \nabla_{v} \zeta\right) \\
- & b \int_{\mathbb{R}^{2 N}} \varepsilon\left(x \cdot \nabla_{x}\left(|x|^{2} \zeta\right)-v \cdot \nabla_{v}\left(|x|^{2} \zeta\right)\right. \\
+ & \int_{\mathbb{R}^{2 N}}|x|^{2} \varepsilon E_{\varepsilon} \cdot \nabla_{v} \zeta .
\end{aligned}
$$

First observe from (2.33) and an explicit computation that

$$
\int_{\mathbb{R}^{2 N}}\left(x \cdot \nabla_{x} Q_{b}-v \cdot \nabla_{v} Q_{b}\right)|x|^{2}=2 \int_{\mathbb{R}^{2 N}}|x|^{2} Q(1+o(1)) .
$$

The other terms in $\varepsilon$ are estimated using the fact that $\zeta$ is compactly supported in $(x, v)$, and we eventually get

$$
\left|\frac{\lambda_{s}}{\lambda}+b\right| \leq C\left[\left|b_{s}\right|+\int_{\mathbb{R}^{2 N}}\left(1+|v|^{2}\right)|\varepsilon|+\left|E_{\varepsilon}\right|_{L^{2}}\right],
$$

and (3.73) and (3.74) now imply (3.75) for $N=4$.

Case $N=3$ : The equation for $g$ is now

$$
\partial_{s} g+\frac{v}{\sqrt{\lambda^{2}+|v|^{2}}} \cdot \nabla_{x} g-E_{g} \cdot \nabla_{v} g=\frac{\lambda_{s}}{\lambda}\left(x \cdot \nabla_{x} g-v \cdot \nabla_{v} g\right) .
$$


Expanding $g=Q_{b}+\varepsilon$ and using (1.21) yields

$$
\begin{aligned}
& b_{s} \frac{\partial Q_{b}}{\partial b}+\partial_{s} \varepsilon+\frac{v}{\sqrt{\lambda^{2}+|v|^{2}}} \cdot \nabla_{x} \varepsilon-E_{Q_{b}} \cdot \nabla_{v} \varepsilon-E_{\varepsilon} \cdot \nabla_{v} Q_{b} \\
+ & b\left(x \cdot \nabla_{x} \varepsilon-v \cdot \nabla_{v} \varepsilon\right)-E_{\varepsilon} \cdot \nabla_{v} \varepsilon \\
= & \left(\frac{\lambda_{s}}{\lambda}+b\right)\left(x \cdot \nabla_{x} Q_{b}-v \cdot \nabla_{v} Q_{b}\right)+\left(\frac{\lambda_{s}}{\lambda}+b\right)\left(x \cdot \nabla_{x} \varepsilon-v \cdot \nabla_{v} \varepsilon\right) \\
- & \left(\frac{v}{\sqrt{\lambda^{2}+|v|^{2}}}-\frac{v}{|v|}\right) \cdot \nabla_{x} Q_{b} .
\end{aligned}
$$

We take the inner product of (3.76) with $|v||x|^{2} \zeta$. We first have from explicit computation

$$
\int_{\mathbb{R}^{2 N}}\left(x \cdot \nabla_{x} Q_{b}-v \cdot \nabla_{v} Q_{b}\right)|x|^{2}|v|=-\int|x|^{2}|v| Q(1+\delta(b(0))) .
$$

All the other terms in $\varepsilon$ are estimated as for $N=4$ up to the corrective terms in $Q_{b}$ which are estimated by integrating by parts:

$$
\begin{aligned}
& \left.\left|\int_{\mathbb{R}^{2 N}}\right| v|| x\right|^{2} \zeta\left(\frac{v}{\sqrt{\lambda^{2}+|v|^{2}}}-\frac{v}{|v|}\right) \cdot \nabla_{x} Q_{b} \mid \\
& \quad \leq C \int_{\mathbb{R}^{2 N}}|v|\left|\frac{v}{\sqrt{\lambda^{2}+|v|^{2}}}-\frac{v}{|v|}\right| Q_{b} \leq C \sqrt{\lambda} \leq C b^{2} .
\end{aligned}
$$

We thus again estimate

$$
\left|\frac{\lambda_{s}}{\lambda}+b\right| \leq C\left[\left|b_{s}\right|+\int_{\mathbb{R}^{2 N}}(1+|v|)|\varepsilon|+\left|E_{\varepsilon}\right|_{L^{2}}\right],
$$

and (3.73) and (3.74) now imply (3.75) for $N=3$.

Step 3. Conclusion.

Let us now conclude the proofs of (3.16) and (3.18) which are a consequence of (3.75). Indeed, first observe that (3.75) and (3.17) imply

$$
\frac{\lambda_{s}}{\lambda} \leq-\frac{b(0)}{2} \leq 0
$$

and (3.16) follows. Integrating this in time yields

$$
\lambda(s) \leq \lambda(0) e^{-\frac{b(0)}{2}\left(s-s_{0}\right)} \leq e^{-\frac{b(0) s}{2}}
$$

from (3.3), and (3.18) is proved

This concludes the proof of Lemma 3

3.5. Proof of Theorem 3. We are now in a position to prove Theorem 3 ,

Step 1. Finite time blow up and control of the speed.

Let an initial data $f_{0}$ be as in the hypothesis of Theorem 3 and $f(t)$ the corresponding classical solution to (1.3) for $N=3$ or (1.14) for $N=4$, with $[0, T)$, $0<T \leq+\infty$ its maximum time interval existence. 
The continuity of the flow $f \in \mathcal{C}([0, T), \mathcal{E})$ and the bootstrap Lemma 3 imply from a continuation argument that $f(t)$ admits for all $t \in[0, T)$ a nonlinear decomposition (3.1), and the estimates (3.15), (3.16), (3.17) and (3.18) hold for all $t \in[0, T)$. In particular, we have from (3.2) and (3.18)

$$
T \leq s_{0}+\int_{s_{0}}^{+\infty} \lambda^{N-2}(s) d s \leq C(b(0))+\int_{1}^{+\infty} e^{-\frac{(N-2) b(0) s}{2}} \leq C(b(0))<+\infty,
$$

and thus the solution blows up in finite time $T<+\infty$. The local well-posedness theory provided by Theorem 1 now implies

$$
\left.\left.|| v\right|^{N-2} f(t)\right|_{L^{1}} \rightarrow+\infty \text { as } t \rightarrow T \text {. }
$$

On the other hand, (3.15) and (3.24) imply

$$
\frac{1}{C} \leq\left.\left.\lambda^{N-2}(t)|| v\right|^{N-2} f(t)\right|_{L^{1}} \leq C
$$

and thus

$$
\lambda(t) \rightarrow 0 \text { as } t \rightarrow T .
$$

Now from (3.75), we have: $\forall t \in[0, T)$,

$$
\frac{b(0)}{2} \leq-\frac{\lambda_{s}}{\lambda}=\frac{1}{N-2} \frac{d}{d t} \lambda^{N-2} \leq 2 b(0) .
$$

We integrate this in time using (3.78) to derive

$$
\frac{b(0)(T-t)}{2} \leq \lambda^{N-2}(t) \leq 2 b(0)(T-t)
$$

which together with (3.77) implies the estimate on the blow up speed (1.28).

Step 2. Dispersive control outside the singularity.

We now turn to the proof of the gain of regularity outside the origin (1.29) which is a consequence of the dispersive virial estimate (3.21).

Let $\lambda_{f}$ and $\tilde{f}$ be given by (1.26), (1.27):

$$
\tilde{f}(t, x, v)=f(t, x, v)-Q\left(\frac{x}{\lambda_{f}(t)}, \lambda_{f}(t) v\right), \quad \lambda_{f}^{N-2}(t)=\frac{\left.\left.|| v\right|^{N-2} Q\right|_{L^{1}}}{\|\left.\left. v\right|^{N-2} f(t)\right|_{L^{1}}} .
$$

We claim the following dispersive control:

$$
\forall R>0,\left.\left.\quad \int_{0}^{T}|| v\right|^{N-2} \tilde{f}(t)\right|_{L^{1}(|x|>R)} d t<C(R) .
$$

Proof of (3.79). It is a consequence of the viriel dispersive virial estimate (3.21). Let us first recall the geometrical decomposition (3.1):

$$
f(t, x, v)=\left(Q_{b(t)}+\varepsilon\right)\left(t, \frac{x}{\lambda(t)}, \lambda(t) v\right) .
$$

Observe from (1.26) and the uniform smallness estimate on $\varepsilon$ (3.15) that

$$
\forall t \in[0, T), \quad \frac{1}{2} \leq \frac{\lambda(t)}{\lambda_{f}(t)} \leq 2 .
$$


Now pick $R>0$; then from $\lambda(t) \rightarrow 0$ as $t \rightarrow T$ and the compact support of $Q$ and $Q_{b(t)}$, the decompositions (1.27) and (3.1) imply that there exists $t(R) \in[0, T)$ such that

$$
\forall t \in[t(R), T), \quad \forall|x|>R, \quad f(t, x, v)=\varepsilon\left(t, \frac{x}{\lambda(t)}, \lambda(t) v\right)=\tilde{f}(t, x, v) .
$$

We now claim that (3.21) implies

$$
\int_{s_{0}}^{+\infty} \int_{|x||v| \geq C}|v|^{N-2} \varepsilon(s, x, v) d s<C\left(f_{0}\right)
$$

for some universal constant $C>0$. Indeed, the definition of $\psi(2.39)$ and the virial dispersive estimate (3.21) yield for some universal constant $C>0$ and all $s \geq s_{0}$ :

$$
C \int_{|x||v| \geq C}|v|^{N-2} \varepsilon(s, x, v)<C\left(f_{0}\right) \sqrt{\lambda}-\frac{d}{d s}\left(\int_{\mathbb{R}^{2 N}}(x \cdot v) Q_{b}\right) .
$$

We now integrate this from $s_{0}$ to $\infty$. The first term is bounded from (3.18) which implies

$$
\int_{s_{0}}^{+\infty} \sqrt{\lambda(s)} d s \leq C \int_{1}^{+\infty} e^{-\frac{b(0)}{4} s} \leq C(b(0))
$$

while for the second term

$$
\left|\int_{s_{0}}^{s_{1}} b_{s}(s) d s\right| \leq C(b(0))
$$

from (3.14), and (3.81) is proved.

In order to prove (3.79), we now observe from (3.80) and (3.2) that

$$
\begin{aligned}
& \left.\left.\int_{t(R)}^{T}|| v\right|^{N-2} f(t)\right|_{L^{1}(|x|>R)} d t=\int_{s(t(R))}^{+\infty} \int_{|x| \geq \frac{R}{\lambda(s)}}|v|^{N-2} \varepsilon(s, x, v) d x d v d s \\
\leq & \int_{s_{0}}^{+\infty} \int_{|x||v| \geq C}|v|^{N-2} \varepsilon(s, x, v) d s+C(R) \int_{s_{0}}^{+\infty} \lambda^{N-2}(s)|\varepsilon(s)|_{L^{1}} d s \\
< & C\left(f_{0}\right)+C(R) T
\end{aligned}
$$

where we used the uniform $L^{1}$ bound on $\varepsilon$ from (3.15), (3.81) and (3.2) again in the last step. This concludes the proof of (3.79).

Step 3. Proof of (1.29).

Let a smooth radially symmetric cut-off function

$$
\xi_{R}(x)=\xi\left(\frac{x}{R}\right) \text { with } \xi(x)=\left\{\begin{array}{lll}
0 & \text { for } & |x| \leq 2, \\
1 & \text { for } & |x| \geq 3
\end{array}\right.
$$

and let

$$
h_{R}(t, x, v)=(1+|v|) \xi_{R}(x) f(t, x, v), \quad E_{R}(t, x)=\xi\left(\frac{2 x}{R}\right) E_{f}(t, x) .
$$

Let us consider (1.14) for $N=4$. Then $h_{R}$ satisfies the equation

$$
\partial_{t} h_{R}+v \cdot \nabla_{x} h_{R}-E_{R} \cdot \nabla_{v} h_{R}=(1+|v|) v f \cdot \nabla_{x} \xi_{R}-E_{R} \cdot \frac{v}{|v|} \xi_{R} f \text { for (1.14) } .
$$


From the regularity $f \in \mathfrak{C}^{0}\left([0, T), \mathcal{C}_{0, \text { rad }}^{1}\right)$, we may consider the characteristic trajectories $X(s ; t, x, v), V(s ; t, x, v)$ for any initial time $t \in[0, T)$ :

$$
\left\{\begin{array}{l}
\dot{X}(s)=V(s), \quad \dot{V}(s)=-E_{R}(s, X(s)) \\
X(t ; t, x, v)=x, \quad V(t ; t, x, v)=v
\end{array}\right.
$$

Remark from the radial assumption, (2.38) and the definition (3.82) that

$$
\left|E_{R}\right|_{L^{\infty}\left([0, T], L^{\infty}\left(\mathbb{R}^{4}\right)\right)}<C
$$

and thus the characteristic curves are globally Lipschitz continuous in time: $\forall\left[s, t, t^{\prime}\right]$ $\in[t, T)^{2}$,

$$
\left|X(s ; t, x, v)-X\left(s ; t^{\prime}, x, v\right)\right|+\left|V(s ; t, x, v)-V\left(s ; t^{\prime}, x, v\right)\right| \leq C\left|t-t^{\prime}\right| .
$$

In particular, they may be uniquely continued at $t=T$. We then rewrite (3.82) in Duhamel form: $\forall t \in[0, T)$,

$h_{R}(t, x, v)=h_{R}(0, X(0 ; t, x, v), V(0 ; t, x, v))+\int_{0}^{t} Z(s, X(s ; t, x, v), V(s ; t, x, v)) d s$,

with

$$
Z(t, x, v)=(1+|v|) v f \cdot \nabla_{x} \xi_{R}-E_{R} \cdot \frac{v}{|v|} \xi_{R} f .
$$

The regularity of the initial data $f_{0}$ and the Lipschitz smoothness of the characteristic curves ensures

$h_{R}(0, X(0 ; t, x, v), V(0 ; t, x, v)) \rightarrow h_{R}(0, X(0 ; T, x, v), V(0 ; T, x, v))$ in $L^{1}$ as $t \rightarrow T$.

We now claim

$$
\int_{0}^{t} Z(s, X(s ; t, x, v), V(s ; t, x, v)) d s \text { has a strong } L^{1} \text { limit as } t \rightarrow T,
$$

which concludes the proof of (1.29) for (1.14) in the case $N=4$.

Proof of (3.85). Pick $\varepsilon>0$ and let $t_{0} \leq t<T$. Then

$$
\begin{aligned}
& \left|\int_{t_{0}}^{t} Z(s, X(s ; t, x, v), V(s ; t, x, v)) d s\right|_{L^{1}} \\
& \quad \leq \int_{t_{0}}^{t}|Z(s, X(s ; t, x, v), V(s ; t, x, v))|_{L^{1}}=\int_{t_{0}}^{t}|Z(s)|_{L^{1}} d s \\
& \quad \leq C \int_{t_{0}}^{T}\left|\left(1+|v|^{2}\right) f(s)\right|_{L^{1}(|x|>R)} d s<\varepsilon
\end{aligned}
$$

for $t_{0}(\varepsilon)$ close enough to $T$ from (3.79). We also used the fact that the diffeomorphism $(x, v) \rightarrow(X(s ; t, x, v), V(s ; t, x, v))$ preserves the Lebesgue measure. On the other hand,

$$
\left|\int_{0}^{t_{0}}\left[Z(s, X(s ; t, x, v), V(s ; t, x, v))-Z\left(s, X\left(s ; t_{0}, x, v\right), V\left(s ; t_{0}, x, v\right)\right)\right] d s\right|_{L^{1}} \leq \varepsilon
$$

for $\left|t-t_{0}\right|<\delta(\varepsilon)$ from the smoothness of the characteristics and the regularity of $Z$ on $\left[0, t_{0}\right]$. We conclude that $\int_{0}^{t} Z(s, X(s ; t, x, v), V(s ; t, x, v)) d s$ is a Cauchy sequence in $L^{1}$ as $t \rightarrow T$, and (3.85) follows.

The proof for (1.3) for $N=3$ is very similar and left to the reader. This completes the proof of Theorem 3 . 


\section{ACKNOWLEDGMENTS}

Pierre Raphaël would like to thank for its kind hospitality the Laboratoire de Mathematiques pour l'Industrie et la Physique, Universite Paul Sabatier, Toulouse, France, where part of this work was done. Florian Méhats was supported by the ACI Nouvelles Interfaces des Mathématiques no. ACINIM 176-2004 funded by the French ministry of research as well as the ACI Jeunes chercheurs no. JC1035. Moreover, the authors express their gratitude to the referees for numerous comments and new references connected to this work.

\section{REFERENCES}

[1] Batt, J., Global symmetric solutions of the initial value problem of stellar dynamics, J. Differential Equations 25 (1977), no. 3, 342-364. MR0487082 (58:6749)

[2] Batt, J.; Faltenbacher, W.; Horst, E., Stationary spherically symmetric models in stellar dynamics, Arch. Rat. Mech. Anal. 93, 159-183 (1986). MR823117(87i:85001)

[3] Binney, J.; Tremaine, S., Galactic Dynamics, Princeton University Press, 1987.

[4] Bouchut, F.; Golse, F.; Pulvirenti, M., Kinetic equations and asymptotic theory, Series in Appl. Math., Gauthiers-Villars, Paris, 2000. MR2065070 (2005d:82102)

[5] Bourgain, J.; Wang, W., Construction of blowup solutions for the nonlinear Schrödinger equation with critical nonlinearity, Ann. Scuola Norm. Sup. Pisa Cl. Sci. (4) 25 (1997), no. 1-2, 197-215 (1998). MR 1655515 (99m:35219)

[6] Choquet-Bruhat, Y.; Noutchegueme, N., Global solutions of the Yang-Mills-Vlasov system with zero rest mass, C. R. Acad. Sci. Paris Ser. I Math. 311 (1990), no. 12, 785-788. MR 1082633 (92g:58128)

[7] Cote, R., Construction of solutions to the subcritical gKdV equations with a given asymptotic behaviour, J. Funct. Anal. 241 (2006), no. 1, 143-211. MR2264249 (2007h:35279)

[8] Dafermos, M., A note on the collapse of small data self-gravitating massless collisionless matter, J. Hyp. Diff. Eq. 3, 589-598. MR2289606

[9] Dafermos, M.; Rendall, A., An extension principle for the Einstein-Vlasov system under spherical symmetry, Ann. Henri Poincare 6 (2005), 1137-1155. MR2189379 (2007c:83011)

[10] Degond, P., Local existence of solutions of the Vlasov-Maxwell equations and convergence to the Vlasov-Poisson equations for infinite light velocity, Math. Methods Appl. Sci. 8 (1986), no. 4, 533-558. MR870991 (88a:76054)

[11] DiPerna, R. J.; Lions, P.-L., Global weak solutions of Vlasov-Maxwell systems, Comm. Pure Appl. Math. 42 (1989), no. 6, 729-757. MR 1003433 (90i:35236)

[12] Dolbeault, J., Explosion à temps fini et évanescence en temps grand dans les équations cinétiques de la gravitation, unpublished manuscript.

[13] Fibich, G.; Merle, F.; Raphaël, P., Numerical proof of a spectral property related to the singularity formation for the $L^{2}$ critical non linear Schrödinger equation, to appear in Physica D.

[14] Fridmann, A. M.; Polyachenko, V. L., Physics of gravitating systems, Springer-Verlag

[15] Frohlich, J.; Lenzman, E., Dynamical Collapse of White Dwarfs in Hartree- and Hartree-Fock Theory, preprint arXiv/math-ph/0608020.

[16] Frohlich, J.; Jonsson, L.; Lenzman, E., Boson Stars as Solitary Waves, to appear in Commun. Math. Phys., preprint arXiv/math-ph/0512040.

[17] Glassey, R., The Cauchy problem in kinetic theory, Society for Industrial and Applied Mathematics (SIAM), Philadelphia, PA, 1996. MR1379589(97i:82070)

[18] Glassey, R.T.; Schaeffer, J., On symmetric solutions of the relativistic Vlasov-Poisson system, Comm. Math. Phys. 101 (1985), no. 4, 459-473. MR815195 (87c:35138)

[19] Glassey, R. T.; Schaeffer, J. W., Global existence for the relativistic Vlasov-Maxwell system with nearly neutral initial data, Comm. Math. Phys. 119 (1988), no. 3, 353-384. MR969207 (90b:82042) 
[20] Glassey, R. T.; Schaeffer, J., The relativistic Vlasov-Maxwell system in two space dimensions. I, II, Arch. Rational Mech. Anal. 141 (1998), no. 4, 331-354, 355-374. MR1620506 (99d:82071)

[21] Glassey, R. T.; Schaeffer, J., On global symmetric solutions to the relativistic Vlasov-Poisson equation in three space dimensions, Math. Methods Appl. Sci. 24 (2001), no. 3, 143-157. MR $1810884(2001 \mathrm{~m}: 82082)$

[22] Glassey, R. T.; Strauss, W. A., Singularity formation in a collisionless plasma could occur only at high velocities, Arch. Rational Mech. Anal. 92 (1986), no. 1, 59-90. MR816621 $(87 \mathrm{j}: 82064)$

[23] Guo, Y.; Rein, G., Isotropic steady states in galactic dynamics, Comm. Math. Phys. 219 (2001), 607-629. MR1838751 (2002g:85001)

[24] Hadzic, M; Rein; G., Global existence and nonlinear stability for the relativistic VlasovPoisson system in the gravitational case, to appear in Indiana Univ. Math. J.

[25] Krieger, J., Schlag, W., Non-generic blow-up solutions for the critical focusing NLS in 1-d, preprint (2005).

[26] Lemou, M.; Méhats, F.; Raphaël, P., On the orbital stability of the ground states and the singularity formation for the gravitational Vlasov-Poisson system, to appear in Arch. Rat. Mech. Anal.

[27] Lemou, M.; Méhats, F.; Raphaël, P., Structure of the linearized gravitational Vlasov-Poisson system close to a polytropic ground state, to appear in SIAM J. Math. Anal.

[28] Lemou, M.; Méhats, F.; Raphaël, P., Uniqueness of the critical mass blow up solution for the four dimensional gravitational Vlasov-Poisson system, to appear in Ann. Inst. H. Poincaré Anal. Non Linéaire.

[29] Lieb, E.H.; Yau, H.T., The Chandrasekhar theory of stellar collapse as the limit of quantum mechanics, Comm. Math. Phys. 112 (1987), no. 1, 147-174. MR904142 (89b:82014)

[30] Lions, P.-L.; Perthame, B., Propagation of moments and regularity for the 3-dimensional Vlasov-Poisson system. Invent. Math. 105 (1991), no. 2. MR.1115549(92e:35160)

[31] Martin-Garcia, J.; Gundlach, C., Self-similar spherically symmetric solutions of the massless Einstein-Vlasov system, Phys. Rev. D (3) 65 (2002), no. 8. MR1899211 (2002m:83010)

[32] Merle, F.; Raphaël, P., Blow up dynamic and upper bound on the blow up rate for critical nonlinear Schrödinger equation, Ann. Math. 161 (2005), no. 1, 157-222. MR2150386 (2006k:35277)

[33] Merle, F.; Raphaël, P., Sharp upper bound on the blow up rate for critical nonlinear Schrödinger equation, Geom. Funct. Ana 13 (2003), 591-642. MR1995801 (2005j:35207)

[34] Merle, F.; Raphaël, P., On Universality of Blow up Profile for $L^{2}$ critical nonlinear Schrödinger equation, Invent. Math. 156, 565-672 (2004). MR2061329 (2006a:35283)

[35] Merle, F.; Raphaël, P., On a sharp lower bound on the blow up rate for critical nonlinear Schrödinger equation, J. Amer. Math. Soc. 19 (2006), no. 1, 37-90. MR2169042|(2006j:35223)

[36] Merle, F.; Raphaël, P., Profiles and quantization of the blow up mass for critical nonlinear Schrödinger equation, Comm. Math. Phys. 253 (2004), no. 3, 675-704. MR2116733 (2006m:35346)

[37] Pfaffelmoser, K.; Global classical solutions of the Vlasov-Poisson system in three dimensions for general initial data, J. Diff. Eq. 95 (1992), 281-303. MR.1165424 (93d:35170)

[38] Planchon, F.; Raphaël, P., Existence and stability of the log-log blow up dynamics for the $L^{2}$-critical nonlinear Schrödinger equation in a domain, to appear in Ann. Henri Poincare.

[39] Raphaël, P., Stability of the log-log bound for blow up solutions to the critical nonlinear Schrödinger equation, Math. Ann. 331 (2005), 577-609. MR2122541 (2006b:35303)

[40] Raphaël, P., Existence and stability of a solution blowing up on a sphere for an $L^{2}$ supercritical nonlinear Schrödinger equation, Duke Math. J. 134 no. 2 (2006), 199-258. MR2248831

[41] Rein, G.; Rendall, A. D., Global existence of solutions of the spherically symmetric VlasovEinstein system with small initial data. Comm. Math. Phys. 150 (1992), no. 3, 561-583. MR:1204320 (94c:83028)

[42] Schaeffer, J., Global existence of smooth solutions to the Vlasov-Poisson system in three dimensions, Comm. Part. Diff. Eq. 16 (1991), 1313-1335. MR.1132787 (92g:82113)

[43] Schaeffer, J., Steady States in Galactic Dynamics, Arch. Rational, Mech. Anal. 172 (2004), 1-19. MR2048565 (2004m:35263) 
[44] Van Kampen, N.G.; Felderhof, B.V., Theoretical methods in plasma physics, Amsterdam, North Holland, 1967.

[45] Weinstein, M.I., Nonlinear Schrödinger equations and sharp interpolation estimates, Comm. Math. Phys. 87 (1983), 567-576. MR691044 (84d:35140)

CNRS and Université Paul Sabatier, MiP, 118, Route de Narbonne, 31062 Toulouse, FrANCE

IRmaR, Université Rennes 1, Campus de Beaulieu, 35042 Rennes Cedex, France

CNRS and Université Paris-Sud, Orsay, France 